\title{
Experimental Assessment of the Effects of a Porous Double Skin Façade System on Cladding Loads
}

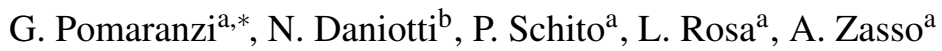 \\ ${ }^{a}$ Politecnico di Milano, Department of Mechanical Engineering \\ via G. La Masa 1, 20156 Milan, Italy \\ ${ }^{b}$ University of Stavanger, Department of Mechanical and Structural Engineering and Material Science \\ N-4036 Stavanger, Norway
}

\section{Abstract}

The high-efficiency façades, such as porous double skins, have become increasingly popular due to the recent technological progress in architecture. The so-called porous double skin façade (DSF) systems, which are constituted by a permeable layer over a closed inner façade, are often adopted to reduce the system energy demand. However, as expected, the porous skin alters the wind-induced pressures acting on the inner façade. Therefore, the cladding loads for such a façade system has to be accurately estimated performing wind tunnel tests. Using the low-rise buildings of the New Bocconi Campus as a case study, we present the experimental wind tunnel methodologies utilized to assess the wind-induced peak pressures acting on the inner glazed skin of the porous double skin façade system designed for the case at hands. In particular, the reduction of both the positive and negative peak pressures estimated for the inner façade is addressed when comparing the standard façade to the porous DSF case. In addition, the valuable data set of the pressure signals acquired for the porous DSF system studied, allows one to investigate the dependence of the computed peak pressures on the averaging time utilized for the extreme value estimates.

${ }^{*}$ Corresponding author. Politecnico di Milano, Department of Mechanical Engineering, via La Masa 1, 20156 Milan, Italy. e-mail: giulia.pomaranzi@polimi.it

Email addresses: giulia.pomaranzi@polimi.it (G. Pomaranzi), nicolo.daniotti@uis.no (N. Daniotti), paolo.schito@polimi.it (P. Schito), lorenzo.rosa@polimi.it (L. Rosa), alberto.zasso@polimi.it (A. Zasso) 
constructed from aluminum, but they can also be made from galvanized steel, cold-rolled steel or stainless steel.

The presence of the outer skin may alter the wind-induced pressure distribution on the inner façade and, thus, the design cladding loads. The expected potential reduction of the design peak pressures compared to the standard glazed façade case, if correctly estimated, has a relevant impact on the economics of the building since the cladding system can achieve up to $25 \%$ of the total building costs (Overend and Zammit. 2006). However, Eurocode and the other National Codes do not currently address the calculation of wind loads when these façade systems are adopted (Kilpatrick et al., 2009). In fact, as pointed by da Silva and Gomes (2008), the impact of the DSF system on the inner skin's wind-induced pressure distribution relevant to the cladding design has been sparsely ascertained, especially when the porosity of the external skin is relatively high, e.g. $10 \div 50 \%$. The porous DSF systems characterized by a porosity lower than $1 \%$ such as rain screens and porous roofs are found to be effective in reducing the wind-induced pressures on the inner façade compared to the standard glazed façade case (Gerhardt and Janser, 1994)(Gerhardt and Kruger, 1997). Belloli et al. (2014) have evaluated the effects of the porosity of perforated steel plates as the cladding of a tall slender tower. The results highlight the peak pressure reduction due to the installation of the permeable elements.

Nowadays the porous structural elements are utilized for civil engineering application also for their aerodynamic behavior. For example, perforated shroudings have been investigated in the wind tunnel as they may represent a suitable device able to control vortex shedding induced vibrations (Belloli et al., 2012). Belloli et al. (2016) have analyzed the aerodynamic behaviour of a cylinder with perforated shrouding, varying the porosity level and the gap between the external mesh and inner surface. Test performed in smooth flow condition and sub-critical Reynolds Number range have highlighted a reduction of vortex shedding induced vibration with the application of the mesh, especially for porosity higher than $40 \%$. Hu et al. (2017) have studied the effects of a double-skin façade with vertical openings installed in front of the windward face on the wind-induced response of the bare CAARC model. The cross-wind response of the structure, associated with vortex shedding phenomena, has been proved to be significantly reduced due to gap flow between the windward face and the inner façade which results in less severe pressure fluctuations acting on the side faces. Kemper and Feldmann (2019) have proposed different wind load recommendations for rectangular shaped buildings with single porous cladding and porous double skin façade. For the latter, the porous cladding elements are made by stainless steel wire meshes with $20 \%$ porosity. Both full-scale measurements and wind tunnel tests in a scaled model have been performed to address the aerodynamic behavior of the porous skin and the corresponding wind loads.

The main scope of this research is to assess the external wind-induced pressure distribution on the inner glazed façade, when an outer porous mesh is installed. The case at hands is the New Bocconi Campus low-rise buildings, the cladding system of which is constituted by a porous DSF, having a diamond-shaped expanded metallic mesh as the outer skin. The multi-scale experimental methods utilized during the wind tunnel tests aim to provide a reliable estimate of the design wind-induced peak pressures acting on the inner façade. To this purpose, the buildings of the case study are first modeled adopting a standard façade system. Thereafter, the metallic mesh is scaled to guarantee the same aerodynamic behaviour of the prototype one, and the wind-induced pressures acquired on the inner skin of the porous DSF system are assessed. Thus, the expected reductions of the peak pressures compared to the standard glazed façade are quantified. The valuable pressure data set is further utilized to study the characteristics of the pressure signals, focusing of the beneficial effects of the adopted outer skin and the impact of the averaging time used in the extreme value analysis.

The paper is organized as follows: Section 2 describes the multi-scale experimental methodologies 


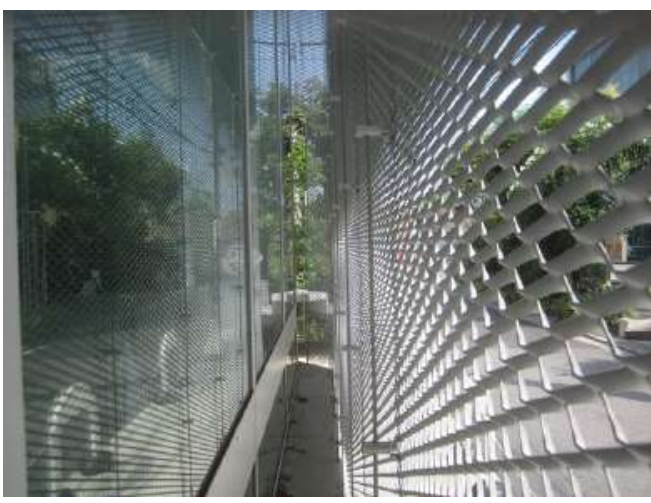

(a)

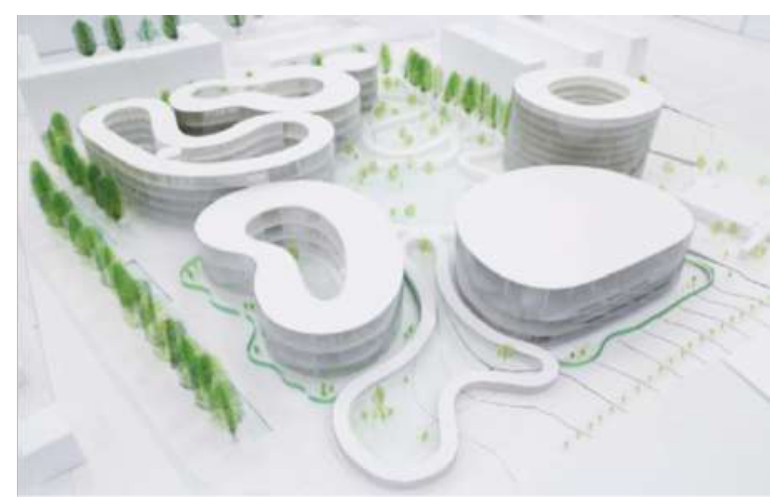

(b)

Figure 1: (a) Example of Double Skin Façade porous system; (b) the New Bocconi Campus rendering.

adopted in the wind tunnel to study a porous DSF system using the New Bocconi Campus low-rise buildings as a case study. Section 3 illustrates the output the pressure measurements performed whereas Section 4 investigates the reduction of the peak pressures on the inner façade.

\section{Wind tunnel tests set-up}

\subsection{Case Study: the New Bocconi Campus buildings}

The New Bocconi Campus is a building complex located within the urban area of $36000 \mathrm{~m}^{2}$ in southern part of Milan (Italy) and comprises six different low-rise buildings, the designated use of which will be offices, dormitories as well as a recreation centre. Figure 1(b) shows a rendering of the structures: they are characterized by cylindrical shape with high curvature radius for the vertical walls. The height of the structures varies from $21 \mathrm{~m}$ to $35 \mathrm{~m}$. The buildings are designed utilizing a double skin porous façade system. The inner glazed façades are covered by a metallic mesh, which thus allows the so-called gap-flow to develop (see Figure 1(a) in between the inner and the outer skin. Figure 2(a) depicts the porous double skin façade designed for the case at hand, whereas Figure 2(b) illustrates the close-up of the prototype outer skin, namely a diamond-shaped expanded metallic mesh. According to the peculiar design of the structures, the perimeter defined by the outer skin does not comply with the one corresponding to the inner glazed façade. In fact, the metallic mesh is bent in the horizontal plane in such a way that the wavy profile shown in Figure 3 is obtained. The distance between the outer and inner skins varies in the range $750 \mathrm{~mm} \div 950 \mathrm{~mm}$ for all the buildings. The basic geometrical characteristics of the mesh are summarized in Table 1 . The listed quantities refer to Figure 4 whereas $k$ is the pressure loss coefficient, which is introduced in section 2.4. The expected 50-years return period mean wind speed at the reference height $H_{r e f}=35 \mathrm{~m}$ (roof height of the tallest building) is computed according to the Eurocode (AFNOR, 2008) assuming terrain category IV, and is equal to $22.5 \mathrm{~m} / \mathrm{s}$.

\subsection{POLIMI Wind Tunnel and Instrumentation}

The experimental activities are conducted in the wind tunnel of Politecnico di Milano, which is is a 1.5 MW closed-circuit wind tunnel, the boundary layer test section of which is $4 \mathrm{~m}$ high, $14 \mathrm{~m}$ wide and $36 \mathrm{~m}$ long. The latter distance is adequate to develop a stable boundary layer whereas the large dimensions of the test section allow one to adopt concurrently a large geometric scale $\lambda_{L}\left(\lambda_{L}=\mathrm{model} / \mathrm{real}\right)$, and a low value 


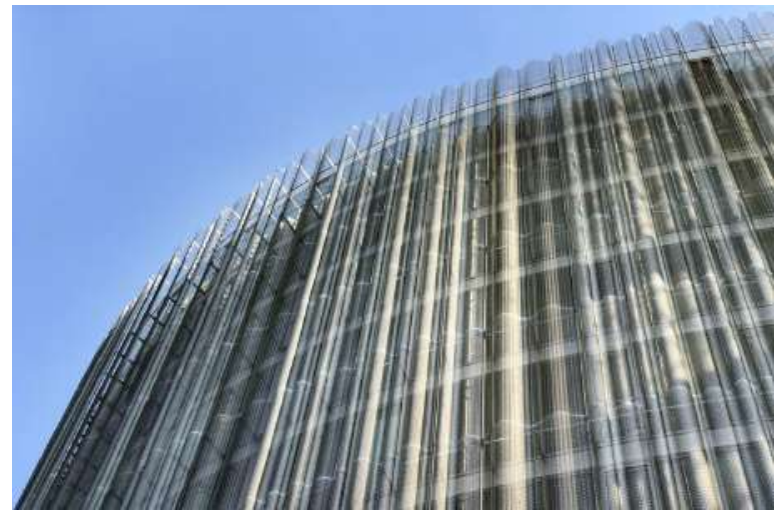

(a)

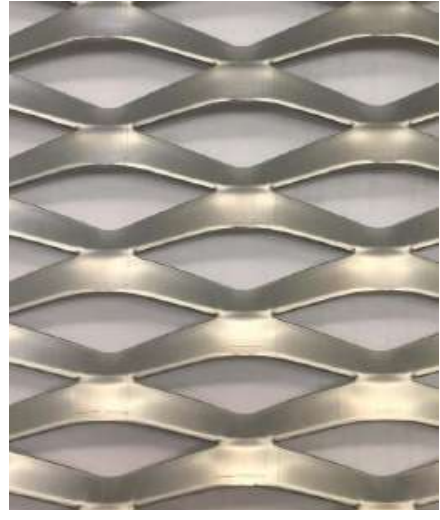

(b)

Figure 2: (a) The double skin with closed inner façade and the outer diamond shaped expanded metallic mesh of the New Bocconi Campus; (b) close-up of the prototype outer mesh.

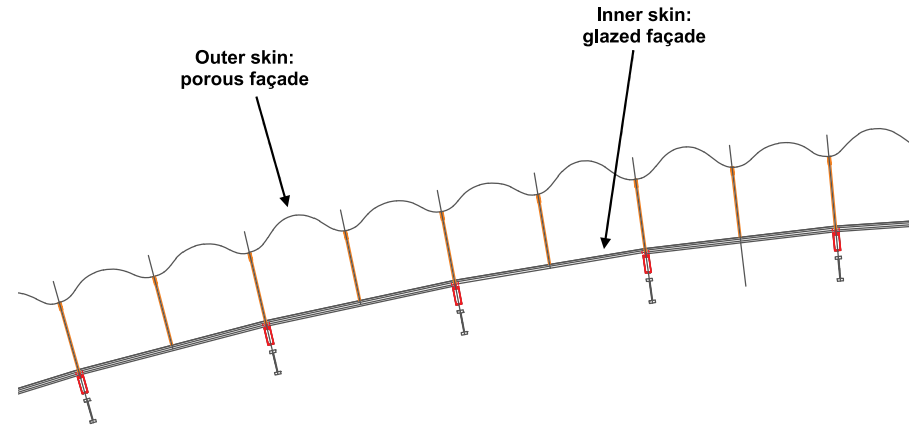

Figure 3: Top view of the Double Skin Façade system: detail of the wavy profile of the outer mesh adopted for the New Bocconi Campus. 
Table 1: Geometrical characteristics of the full-scale and model-scale meshes.

\begin{tabular}{cccc}
\hline & & Full scale & Model scale \\
\hline$D S$ & shorter diagonal & $35 \mathrm{~mm}$ & $3 \mathrm{~mm}$ \\
$D L$ & longer diagonal & $85 \mathrm{~mm}$ & $6 \mathrm{~mm}$ \\
$A v$ & width & $11 \mathrm{~mm}$ & $0.8 \mathrm{~mm}$ \\
$S p$ & thickness & $2 \mathrm{~mm}$ & $0.5 \mathrm{~mm}$ \\
$k$ & pressure loss coefficient & 2.24 & 2.32 \\
\hline
\end{tabular}

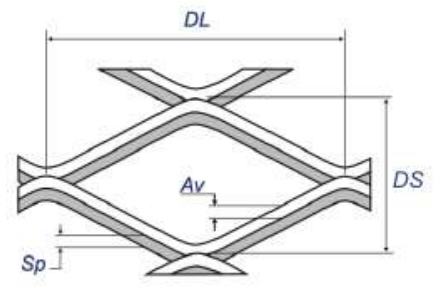

Table 2: The two stages of the wind tunnel tests: name, description, geometric scale $\lambda_{L}$ and velocity scale $\lambda_{V}$.

\begin{tabular}{cccccc}
\hline Stage & Name & Façade System & $\lambda_{L}$ & $\lambda_{V}$ & Sampling time \\
\hline FC1 & Façade Configuration 1 & Standard Glazed Façade & $1: 100$ & $1: 2.53$ & $120 \mathrm{~s}$ \\
FC2 & Façade Configuration 2 & Double Skin Porous Façade & $1: 50$ & $1: 2.14$ & $120 \mathrm{~s}$ \\
\hline
\end{tabular}

of the blockage ratio, which is equal to $1.25 \%$ for the worst case tested. These aspects are fundamental to reduce the effects related to the Reynolds number dependency and avoid flow distortions during the experimental tests, respectively.

To understand the aerodynamic behaviour of the metallic mesh concerning the pressure distribution on the inner façade, this research project is divided into two stages, during which rigid models are tested in turbulent flow conditions. In the first stage, which is referred to as $F C l$ (Façade Configuration 1), cladding loads on rigid models $\left(\lambda_{L}=1: 100\right)$ with no outer skins are assessed. As we model a standard glazed façade system, the main scope of the aforementioned test session is to analyze the peak pressure envelopes of all the buildings and, consequently, identify which building is affected by the most severe cladding loads scenario. The aerodynamic behavior of the outer metallic mesh is adequately modeled before entering the second stage, which is referred to as $F C 2$ (Façade Configuration 2) in the following. In fact, the adequately modelled porous element and the increased geometrical scale $\left(\lambda_{L}=1: 50\right)$ allow one to measure effectively the pressure distributions on both the inner façade and the metallic mesh. Hence, the effects of the porous element on the inner façade's pressures can be investigated.

The models of the building analyzed during both the test sessions are instrumented with 8 PSI ESP-32 HD high-speed pressure scanners, each of them having 32 pressure channels. The scanners are connected to the Initium data acquisition with a sampling frequency of $500 \mathrm{~Hz}$. The sampling time in both stages is $120 \mathrm{~s}$ model scale, which corresponds to 79 minutes and 47 minutes full scale for $F C 1$ and $F C 2$ respectively. The maximum pressure tubing length adopted in the pneumatic chain is kept lower than $90 \mathrm{~cm}$ to minimize the induced distortion in the pressure measurement system. The pressure signals are corrected in the frequency domain, based on the frequency response function estimated according to Bergh and Tijdeman (1965).

\subsection{FCl: set-up and model description}

In the first stage, all five buildings of the New Bocconi Campus are tested. Rigid models, which reproduce the full-scale geometry of the structures, are instrumented with pressure taps (Figure 5(a)). The geometric scale is $\lambda_{L}=1: 100$ whereas the velocity scale is $\lambda_{V}=1: 2.53$, as reported in Table 2. Being the buildings characterized by curved shapes, the Reynolds number dependency is adequately addressed. The external surfaces of the tested buildings are roughened using suitable sandpapers to improve local flow 


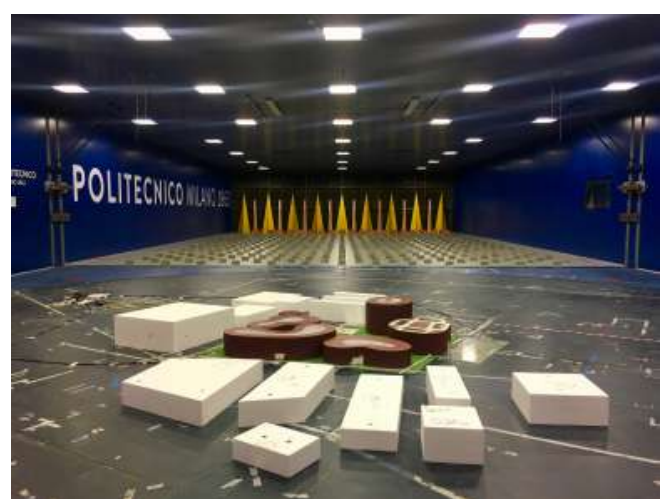

(a)

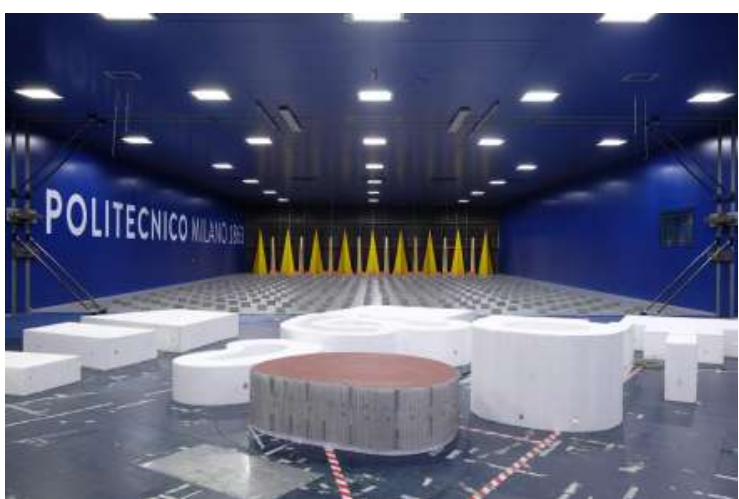

(b)

Figure 5: (a) Set-up of FC1; (b) set-up of FC2.

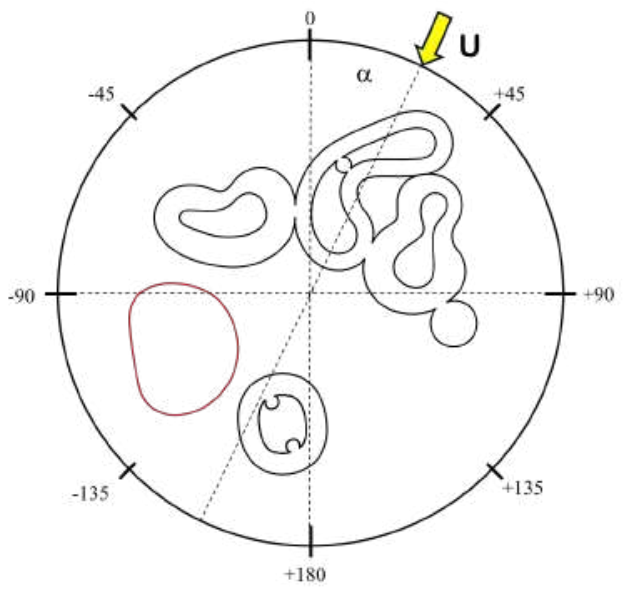

(a)

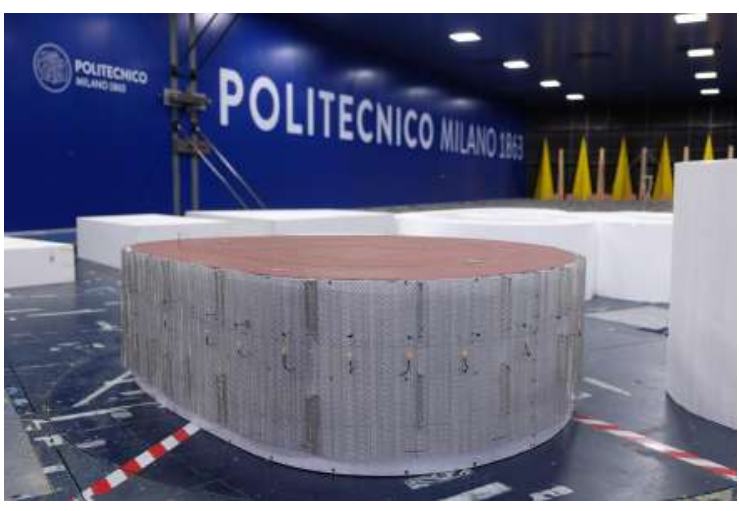

(b)

Figure 6: (a) Wind direction convention and reference system; (b) FC2 building during the second stage of the tests.

similarity and reduce the dependence on the Reynolds number effects. In fact, the surface roughness increases the turbulence locally, allowing one to reproduce the fluid-dynamic effects corresponding to higher Reynolds number. The aforementioned practice is commonly adopted in boundary layer wind tunnel tests when dealing with curved surfaces, see for example Diana et al.(2013). As the structures are low-rise buildings, the pressure taps are distributed along one single level, at $2 / 3$ of the building height with an average spacing of $4 \mathrm{~cm}$ model-scale for each building. The total amount of the measurement points is 256 .

\subsection{Scaling procedure for the permeable façade}

The identification of the porous medium to be used during the FC2 tests is essential to reproduce correctly the aerodynamic behaviour of the double skin façade system. As the air flows through a porous medium, an interaction between the permeable element and the fluid occurs. The geometry of the mesh, its porosity, and the fluid properties are the main parameters the interaction depends on. Specifically, when the flow approaches the porous medium, it passes through narrow openings followed by short section enlargement. This mechanism leads to a pressure drop between the two sides of the permeable element. 


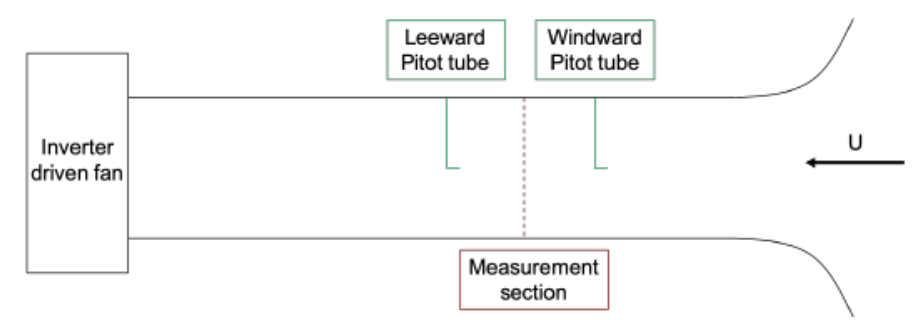

Figure 7: Schematic representation of the open circuit wind tunnel with pressure sensors and location of the porous screen.

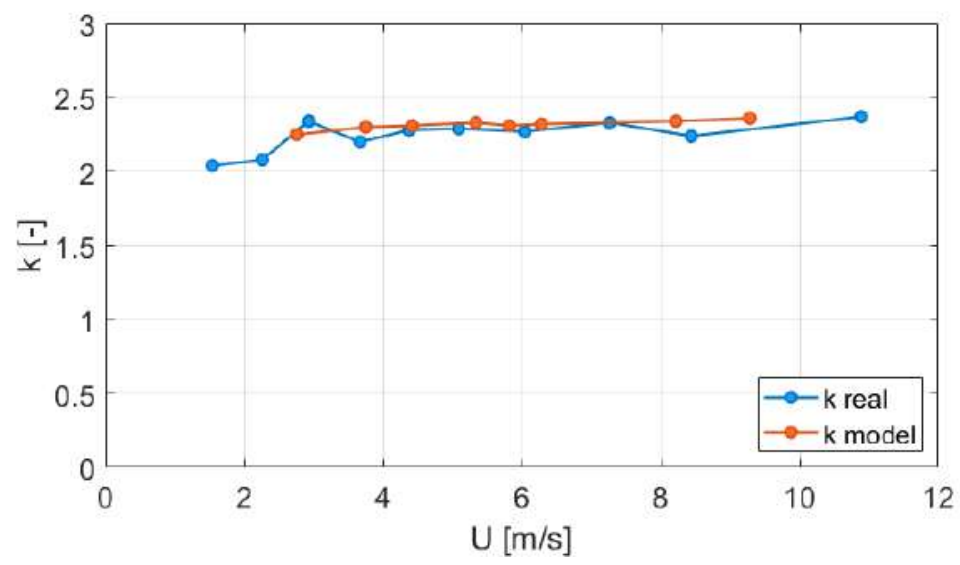

Figure 8: Experimental pressure loss coefficient with varying velocity: real mesh and model mesh.

The geometrical scaling applied to the global structure is not straightforward for those details which are already small at full scale. To ensure the kinematic similitude, e.g. the same flow conditions between real and model scale, the pressure loss coefficient $k$ must be maintained (Belloli et al., 2014) (Letchford et al., 2000). It is defined as:

$$
k=\frac{\Delta p}{\frac{1}{2} \rho U^{2}}
$$

where $\Delta p$ is the pressure difference between the windward and the leeward side of the porous screen; $\rho$ is the air density; $U$ is the mean wind speed. The $k$ parameter is an index of the aerodynamic resistance to the flow through a porous element; it accounts for the effects of porosity and the shape of the holes.

The pressure loss coefficient is experimentally evaluated, performing dedicated tests in a mostly laminar open circuit wind tunnel, with circular cross-section (diameter $400 \mathrm{~mm}$ ). Figure 7 shows a schematic representation of the facility. Two Pitot tubes are positioned at the windward and leeward side of the porous screen, which is placed in the measurement section. Based on the measured pressure values, the pressure loss coefficient is computed through equation 1 .

The $k$ parameter is firstly determined for the real scale mesh. Several samples of mesh models are then tested and the one characterized by the same $k$ value is finally chosen. The pressure loss coefficient comparison of the prototype and model meshes is shown in figure 8; an overall agreement is found between the $k$ parameter curves within the range of wind speed of interest. 


\subsection{FC2: set-up and model description}

The second stage of the research project, e.g. $F C 2$, assesses the pressure distribution on the permeable DSF system, which is adequately reproduced for the building which experiences the highest wind loads during the $F C 1$ wind tunnel tests. The building model equipped with the scaled DSF system is depicted in Figure 5(b) The geometrical scale $\lambda_{L}=1: 50$ is twice as big as $F C 1$ geometrical scale. This enables one to model properly the porous skin, guaranteeing a gap between the two skins large enough to let the gap flow develop. It is worth noting that the velocity scale is $\lambda_{V}=1: 2.14$, as reported in table 2

The model of the building is rigid and the outer porous skin, the characteristics of which are reported in table 1, is installed onto the external surface of the model using rectangular aluminum profiles. The latter guarantee a geometrically scaled distance between the two skins and, at the same time, minimize the potential flow distortion of the gap flow (Figure 9(a)). Before installing it on building model, the mesh is bent to reproduce the wavy profile of the prototype metallic mesh, which can be appreciated from the top view depicted in Figure 3. To measure the pressure distribution on the inner façade, the pressure taps are positioned along the inner perimeter of the building model in such a way that a correspondence between the measurement points of $F C 1$ and $F C 2$ can be established. Additionally, pressure sensors are also placed in columns (Figure 10) to investigate the vertical variation of the wind-induced pressures on the inner skin. The location of such vertically distributed acquisition points is selected based on the most severe wind directions in terms of peak pressures, which are evaluated during $F C 1$ test. Further pressure taps are located along the aforementioned inner perimeter to increase the spatial resolution of the pressure measurements. As previously mentioned, this stage aims also to assess the wind pressure distributions on both the inner façade and the metallic mesh. As the local wind loads on a permeable skin are estimated based on net pressures, the outer skin of the model is instrumented to measure both the external pressure and the inner gap pressure. The former is acquired using pressure taps positioned at the center of a single opening of the mesh previously filled with resin material, trying to minimize the interference between the measurement points distributed on the inner skin. Additionally, pressure taps fixed by small supports (button-like pressure taps shown in Figure 9(a) are mounted onto the external surface of the model mesh to measure the inner gap pressure. The pressure tubing system utilized for the mesh is assumed to have a negligible influence on the pressure signals acquired on the inner façade, given the relatively small outer diameter $(2.4 \mathrm{~mm})$ of the adopted tubes. The total amount of the measurement points during this test is 204 .

Multi-holes cobra probes are utilized to retrieve the 3-components of the velocity of the gap-flow during the FC2 stage. The sensors are installed in the gap between outer and inner skin as well as in front of the external surface of the mesh. Hence, insights into the aerodynamic behaviour of the DSF system complementary to the pressure measurements can be addressed. As the probes are mounted to point towards the expected direction of the oncoming gap-flow, they provide a reliable estimate of the flow fields within a range of $\pm 45^{\circ}$. The adopted sampling frequency is $2000 \mathrm{~Hz}$.

\subsection{Flow Configuration}

As the comparison between the cladding's pressure distribution obtained during $F C l$ and $F C 2$ stages are based on different geometrical scales, an accurate scaling of the atmospheric boundary layer is essential. The latter is adequately modeled based on the characteristic of the site and the surrounding territory. To ensure that the flow conditions agree with the predicted ones, the profiles of the mean wind speed, turbulence intensity and turbulence integral length scale are scaled according to the geometric scale, as shown in Figure 11. It is worth noting that the reference heights $\mathrm{H}_{r e f, j}, j=F C 1, F C 2$ correspond to $35 \mathrm{~m}$ full-scale and the plotted model heights are normalized accordingly. The position of the pressure measurement points are located at 0.4 of $\mathrm{H}_{r e f, j}, j=F C 1, F C 2$ on tested building models. An overall agreement between the profiles 


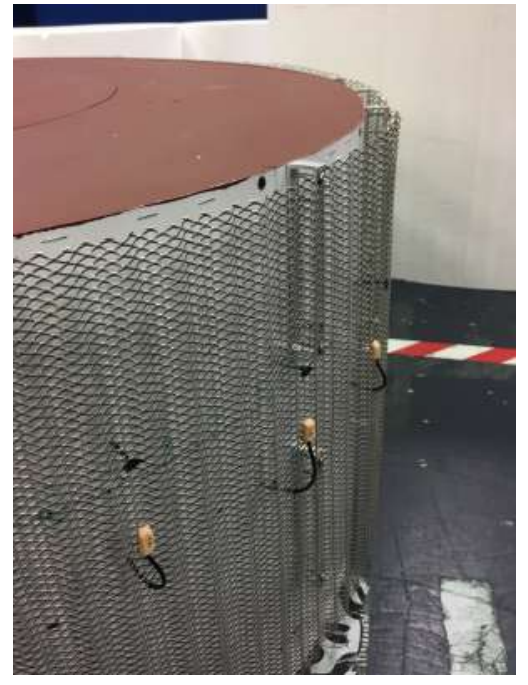

(a)

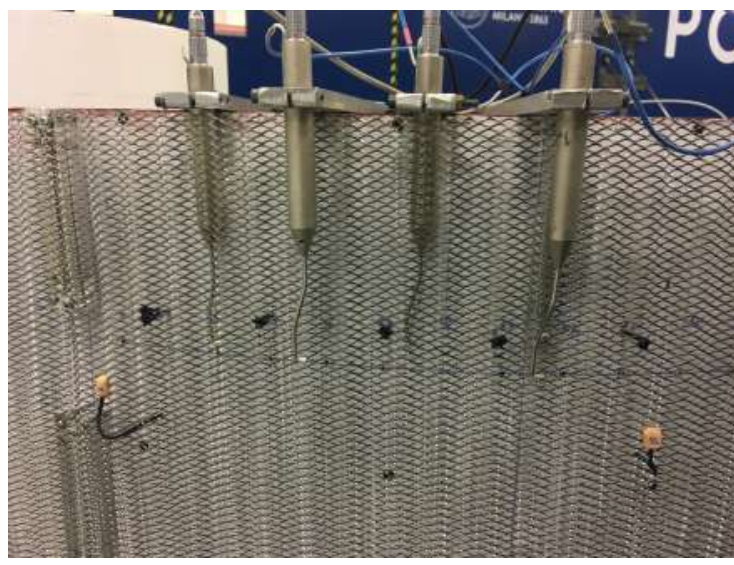

(b)

Figure 9: (a) Close-up insight into the rectangular aluminum profiles used to anchor the outer mesh as well as the measurement system of the net pressure acting on the metallic mesh and (b) Cobra Probes set-up during the $F C 2$ stage.

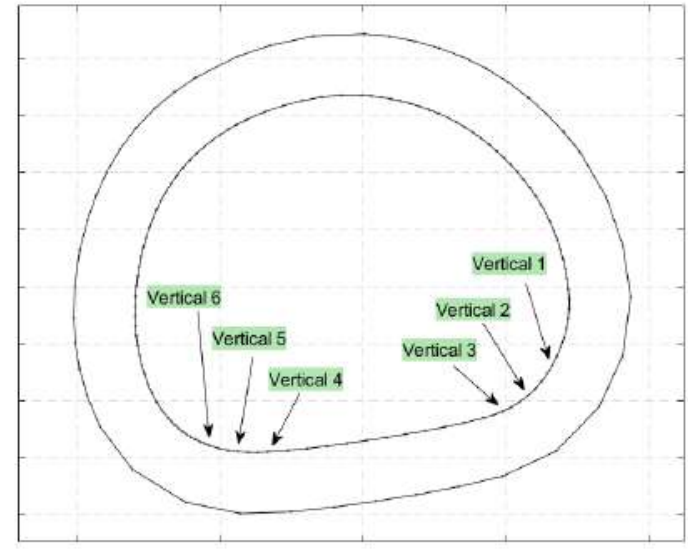

Figure 10: Position of the pressure taps columns on the inner skin of the $F C 2$ model, top view. 
of the mean wind speed normalized by reference mean wind speed at $\mathrm{H}_{r e f, j}, j=F C 1, F C 2$ is found. By fitting the logarithmic profile of the mean wind speed to the measured ones, we obtain a roughness length approximately equal to $1 \mathrm{~m}$.

The profiles' shapes of the along-wind, across-wind, and vertical turbulence intensities are adequately reproduced within the investigated heights of the atmospheric boundary layer. However, the turbulence intensity magnitudes estimated during $F C 2$ are $5 \%$ to $7 \%$ lower than the $F C 1$ ones at $\mathrm{H}_{\text {ref }}$. Discrepancies in the turbulence intensity level may affect the extreme value analysis of the pressure signals and, thus, the estimated peak pressures. However, when it comes to pressures distribution on low-rise buildings, the pressure fluctuations on the façade are also determined by the fluid-structure interactions due to the specific geometry of the surrounding and the shapes of the buildings, which are accurately modeled during the tests. Within the height range of interest, e.g. $z / H_{r e f}<1$, some discrepancies between the turbulent length scale profiles estimated during the tests are found, especially for the along-wind turbulence length scale. However, this is expected since the computation of the auto-correlation function depends on the degree of stationarity of the time histories and, therefore, the resulting turbulence length scale profile is more scattered than other turbulence characteristics. However, as pointed out by Aly and Bitsuamlak (2013), discrepancies on the integral length scale scaling in a wind tunnel test should not affect the prediction of the wind loads for lowrise buildings. Such differences may have an impact on the spatial correlation of the pressure fluctuations, which is out of the scope of the present study.

The agreement between the one-point spectrum of the turbulence components is also addressed, as shown in Figure 12. The one-point spectral density functions normalized by the variance of the velocity are estimated at the reference heights $\mathrm{H}_{r e f, j}, j=F C 1, F C 2$ and compared to Von-Karman velocity spectrum model. The latter spectral model superimposes well to the experimentally estimated velocity spectra.

\section{Experimental Results}

In this section, the wind tunnel tests results are presented. The main aim of this study is the assessment of the impact of the outer permeable skin on the inner façade pressure distribution through a comparison of the wind tunnel tests outcomes. Before proceeding in such comparison, some preliminary ascertainment have to be addressed. Firstly, given the circular shape of the buildings, the Reynolds dependency has to be investigated and discussed properly. Then, it has to be ensured that the different geometric scales are not affecting the results.

\subsection{Pressure Coefficients Definition}

The acquired pressure data are treated in non-dimensional form as local pressure coefficients defined, at the $k$-th pressure tap, as follows:

$$
C_{p}^{k}(t)=\frac{p^{k}(t)-p_{s}}{q_{H}}
$$

where $p^{k}(t)$ is the local pressure, $p_{s}$ is the reference static pressure in the test section and $q_{H}=1 / 2 \rho U_{r e f}^{2}$ is the wind reference dynamic pressure at $\mathrm{H}_{r e f, j}, j=F C 1, F C 2$. From the definition itself, positive pressure loads represent thrusts while negative pressure loads represent suctions (inward and outward arrows respectively in the pressure distributions figures). The pressure coefficients are hereafter presented using their mean value $\left(\bar{C}_{p}\right)$, positive extreme $\left(\hat{C}_{p}\right)$ and negative extreme $\left(\check{C}_{p}\right)$.

When dealing with the metallic mesh, the total wind load acting on the porous skin is computed as the net pressure coefficient, namely the difference in the time domain between the external (ext) and internal (int) pressure coefficients acquired at the $k$-th tap: 


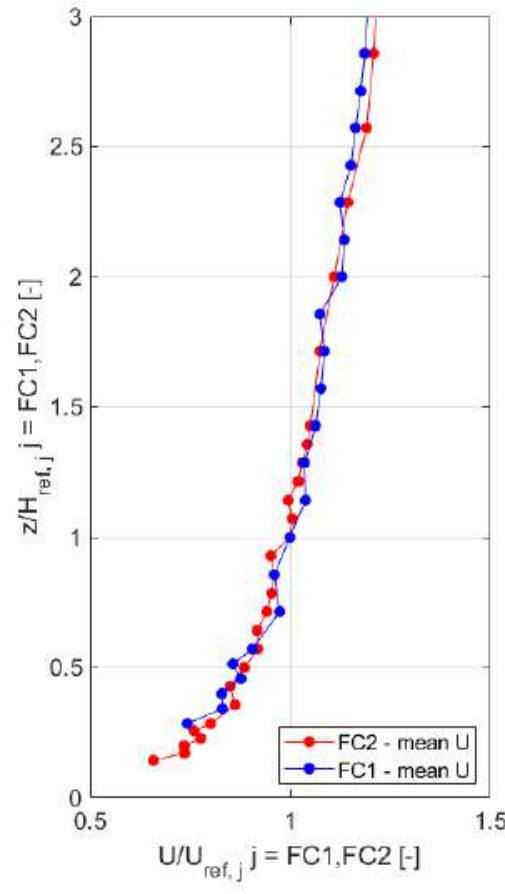

(a)

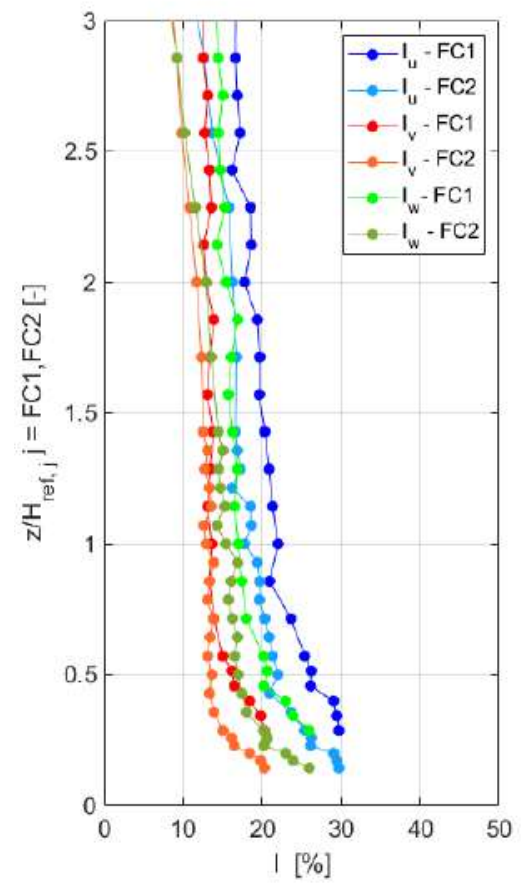

(b)

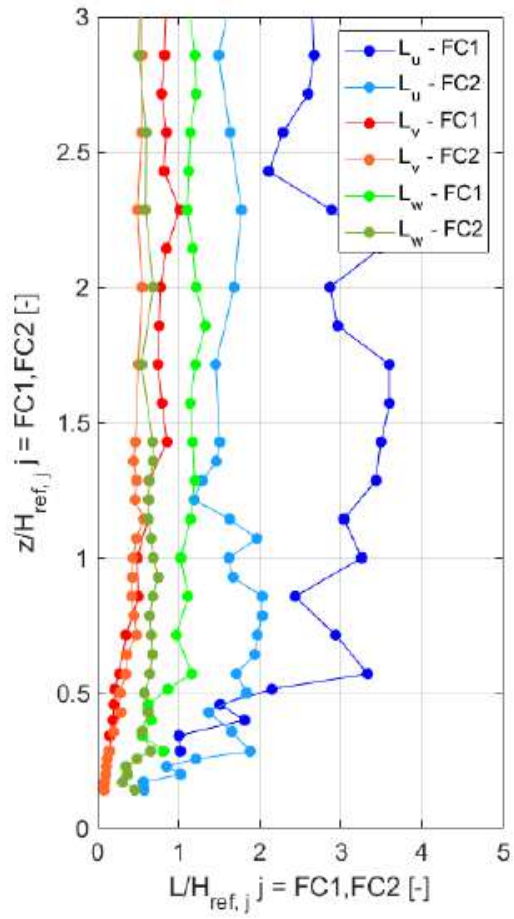

(c)

Figure 11: (a) mean velocity profile, (b) turbulence intensity profile and (c) integral length scales for the $F C 1$ and $F C 2$ tests.

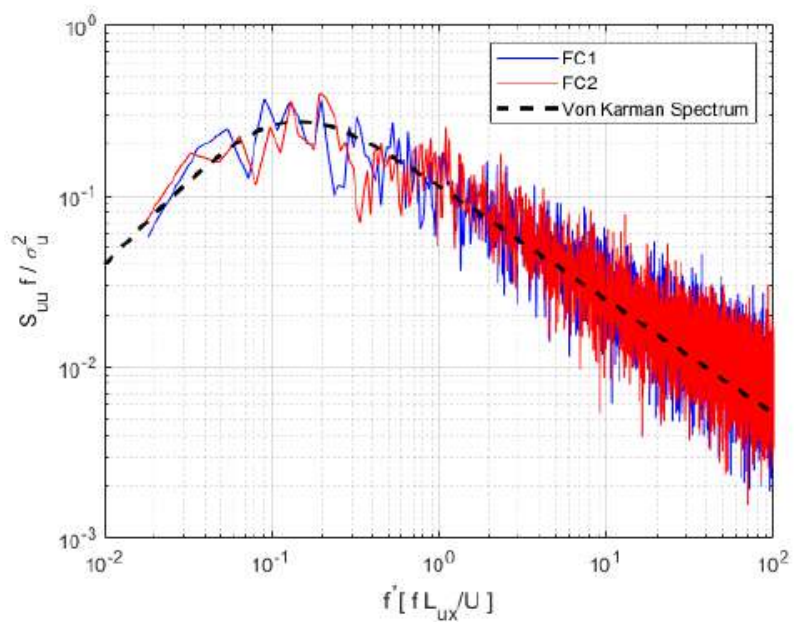

Figure 12: One-point velocity spectral density estimates of the along-wind turbulence at $\mathrm{H}_{r e f, j}, j=F C 1, F C 2$. 
Table 3: Reference wind velocities to test Reynolds dependency. Values are in $\mathrm{m} / \mathrm{s}$.

\begin{tabular}{ccc}
\hline & $F C 1$ & $F C 2$ \\
\hline $\mathrm{U}_{1}\left(H_{\text {ref }}\right)$ & 4.61 & 7.68 \\
$\mathrm{U}_{2}\left(H_{\text {ref }}\right)$ & 6.76 & 8.11 \\
$\mathrm{U}_{3}\left(H_{\text {ref }}\right)$ & 8.57 & 10.01 \\
$\mathrm{U}_{4}\left(H_{\text {ref }}\right)$ & 9.34 & 11.42 \\
\hline
\end{tabular}

$$
C_{p, \text { net }}^{k}(t)=C_{p, \text { ext }}^{k}(t)-C_{p, \text { int }}^{k}(t)
$$

Cladding pressure time-histories are recorded and statistically analyzed to estimate the extreme pressure coefficients (positive and negative) by applying the method introduced by J. Cook and J.R. Mayne (Cook, 1986). Each $C_{p}(t)$ time history is divided into 10 windows, the extreme positive/negative pressure coefficient is extracted from each window and, finally, the Gumbel distribution is fitted to the extreme values (Cook, 1986). Providing that the sampling time is longer than the shortest practicable period of 10 minutes full-scale (Cook and Mayne, 1979) (Mayne and Cook, 1980), the method allows on to correctly estimate the peak pressure coefficient. For the present case, FC1 and FC2 acquisition period corresponds to 79 minutes and 47 minutes full-scale respectively. Hence, the method can be confidently applied and the differences in time history duration do not affect the extreme value estimation.

According the current practice in wind engineering design façade, before proceeding with the extreme value analysis, a low-pass moving average filter with an averaging time equal to $3 \mathrm{~s}$ real scale (the so-called $3 s$ gust $)$ is applied to the signals to account for the lack of correlation of the wind gusts over large areas in such a way that the effects of the eddies smaller than the loaded area are removed.

\subsection{Reynolds number dependency}

As the Reynolds number similitude requirement cannot be achieved in the boundary layer wind tunnel tests and the models are characterized by curved surfaces, the corresponding scaling effects are addressed. In particular, the dependency of the mean pressure coefficients upon the tested mean wind speed is analyzed to assess the sensitivity of the experiments to the Reynolds number. For both the test sessions, four different wind speeds measured at the corresponding $\mathrm{H}_{r e f, j}, j=F C 1, F C 2$ are considered, as reported in table 3 , Based on the maximum mean wind speed considered, the tested Reynolds number are $R e_{F C 1}=3.2 \times 10^{5}$ and $R e_{F C 2}=7.9 \times 10^{5}$. Figure 13 reports the mean pressure coefficients $\bar{C}_{p}$ of 32 pressure taps randomly selected during $F C 1$ (Figure $13(\mathrm{a})$ ) and $F C 2$ tests (Figure 13(b)).

Within the considered wind speed range, for both the test sessions the mean pressure coefficients exhibit a negligible discrepancy when varying the oncoming mean wind speed. Such a result underpins the independence of the acquired pressure signals on the Reynolds number for the tested mean wind speed range and thus make it possible to proceed confidently with the wind tunnel test.

\subsection{Vertical pressure distribution}

The main scope of this research is to deeper into the wind pressure distribution of the inner glazed façade when an outer permeable skin is installed. The research structure, which consists of two different test stages, allows one to compare the pressure distribution from $F C 1$ with the one acquired on the inner cladding in the $F C 2$. Before proceeding in that, it has to be ensured that differences in wind-induced pressure are not due to different geometric scales of the two stages that are responsible for some discrepancies in the boundary layer characteristics, as already pointed out in section 2.6: the turbulence intensities profiles show differences up 


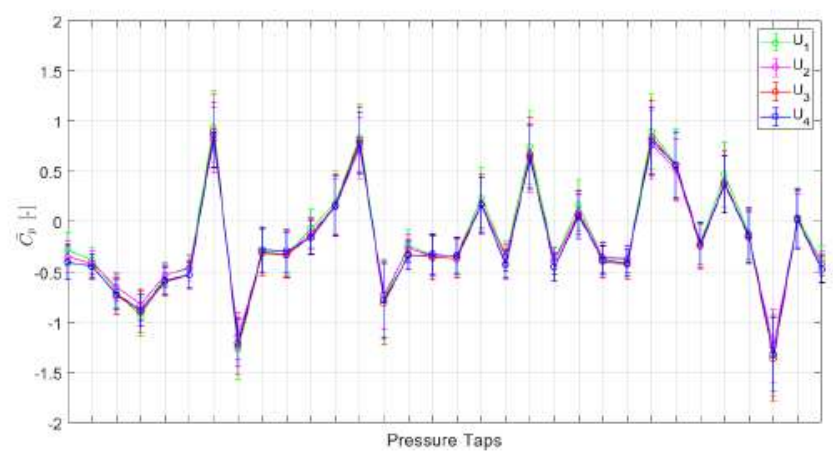

(a)

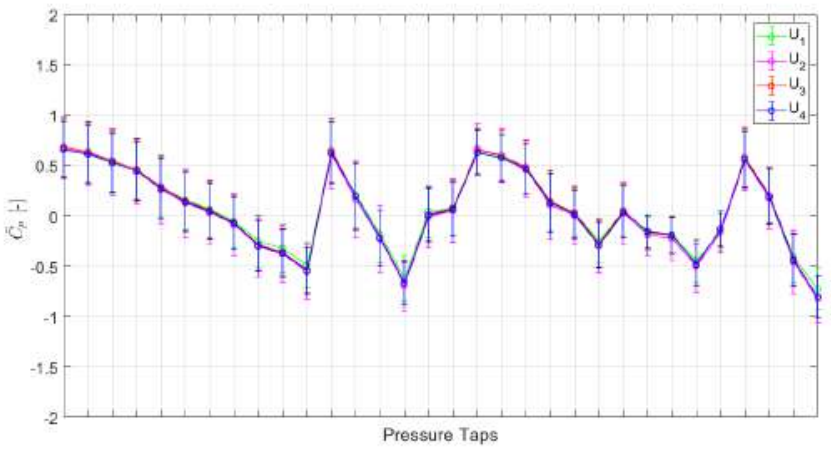

(b)

Figure 13: $\overline{C_{p}}$ dependency on test wind speed for (a) $F C 1$ and (b) $F C 2$ tests. 


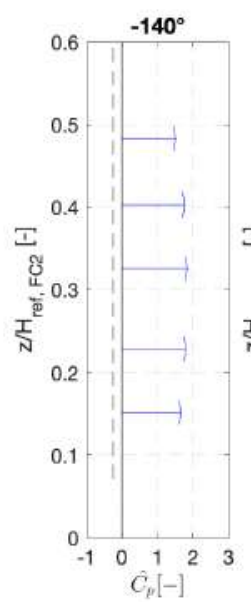

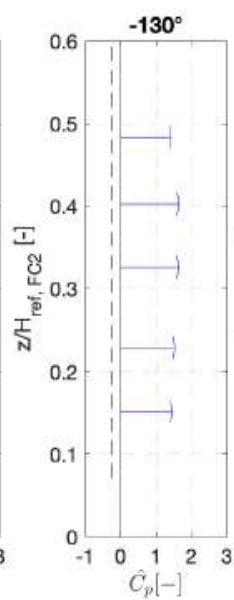

(a)
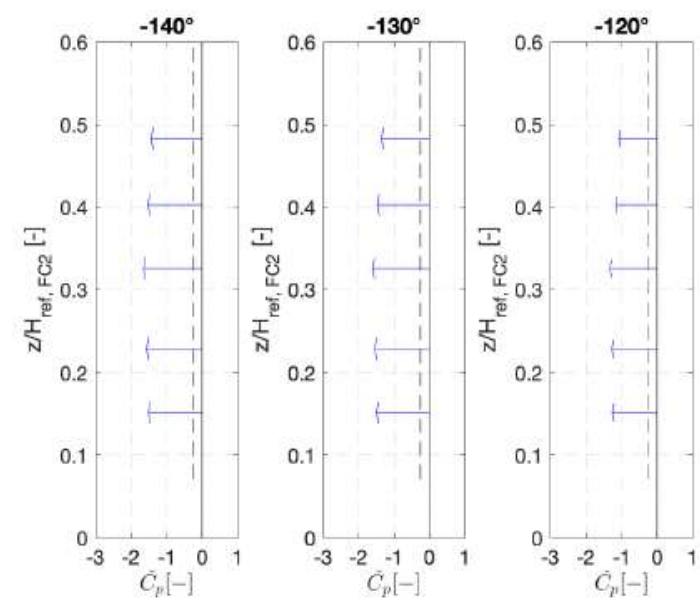

(b)

Figure 14: (a) $\hat{C_{p}}$ distribution for the vertical 2 and (b) $\check{C}_{p}$ distribution for the vertical $5,-140^{\circ},-130^{\circ}$ and $-120^{\circ}$ wind directions.

to $5 \%$ between the $\mathrm{FC} 1$ and $\mathrm{FC} 2$ stages at the same $z$ coordinate while running along the building height, turbulence intensities vary more than $8 \%$.

The pressure sensors placed in columns during the $F C 2$ session (as depicted in Figure 10 allows the possibility to investigate the vertical distribution of the peak pressure coefficients in the inner façade and to discuss their eventual dependency on the turbulence intensity components.

For example, Figure 14 shows the $\hat{C}_{p}$ from Vertical 2 and $\breve{C}_{p}$ from Vertical 5 for the wind directions $-140^{\circ},-130^{\circ}$ and $-120^{\circ}$; a little dependency on the $z$ coordinate of the peak pressure coefficient is found. In particular, the positive and negative peak pressures show the largest values at half height for all the considered exposures. These findings demonstrate that the presence of the outer skin makes the dependency of the extreme values on the height (and so on the turbulence intensities) less severe. This is the main reason why discrepancies in turbulence intensities between the two stages are assumed to not significantly affect the pressure distribution comparison, which can be thus confidently performed.

\subsection{FC1 and FC2 results comparison}

Figure $15(\mathrm{a})$ shows the mean pressure distribution for wind direction $-140^{\circ}$ (Figure $6(\mathrm{a})$ ) from the $F C 2$ test: the inner ring represents the pressure acting on the glazed façade, the outer one refers to the net pressures on the metallic mesh. It emerges that the net pressure on the mesh is highly correlated with the external pressure distribution on the inner façade, even if the former denotes lower values of $C_{p}$ (note that the scale factor for the mean pressure on the mesh is doubled with respect to the one for the inner façade).

The high correlation and the sign of the net pressure on the porous external layer is worth of comments and additional studies. At this aim, by linking the velocity measurements (that will be presented in section 3.5p and pressure distributions, the following mechanism can be highlighted:

- intake with a positive mean net pressure load on the outer skin, coherently with a drop in the velocity component passing from outside to the inner gap shown by the Cobra measurements;

- flow inside the skins gap: the air flows along an ideal line parallel to the inner façade. The flow recorded along the outward normal is found to be negligible compared to the inner façade and there- 


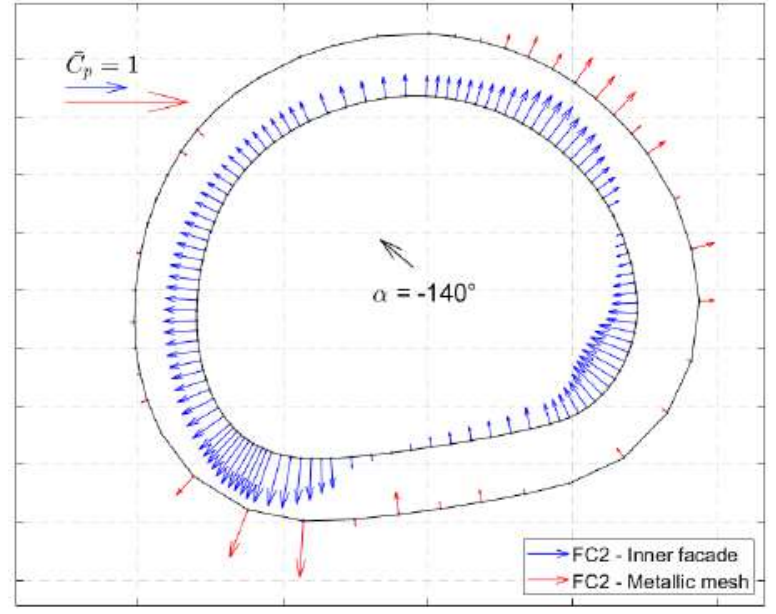

(a)

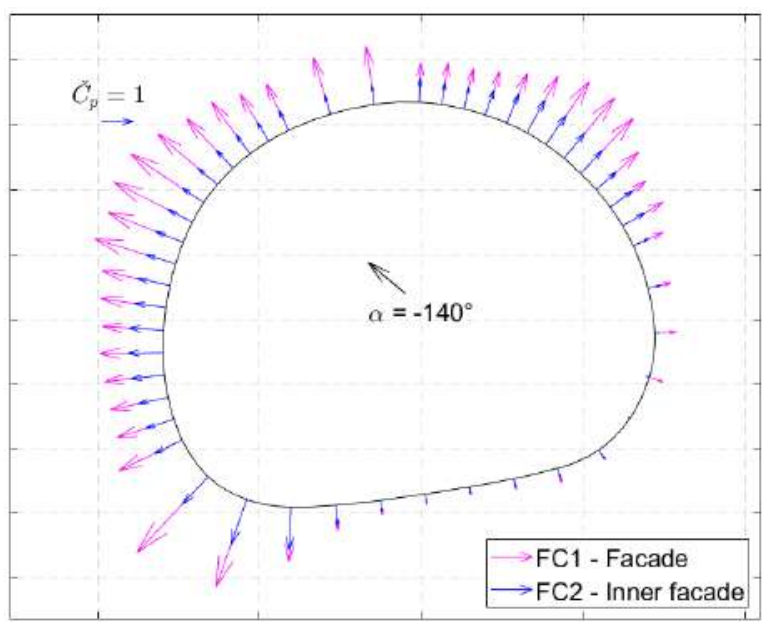

(b)

Figure 15: (a) $\overline{C_{p}}$ distribution for wind direction $-140^{\circ}, F C 2$ test. (b) $\check{C_{p}}$ distribution comparison between $F C 1$ vs $F C 2,-140^{\circ}$ wind direction.

fore, as confirmed by the pressure measurements, a negligible pressure gradient arises between the inward part of the mesh and the façade;

- exit at the large external suction (both on the inner and the outer skin).

Figure $15(\mathrm{~b})$ compares the peak pressure distributions for the FC1 and FC2 tests on the glazed façade: focusing on the negative peak pressures, strong suctions recorded during the $F C 1$ test do not occur in the $F C 2$ system. The outer porous skin beneficial effect is well demonstrated by the envelopes diagrams of the peak pressure coefficients as well, shown in figure 16: the filtering effect holds true for both positive and negative peak pressures.

\subsection{The Effect of the Mesh}

\subsubsection{Cobra Probe measurements}

The main issue involved in the Cobra Probe measurements is associated with the dependency of the accuracy on turbulence levels: the measurements remain relatively accurate for turbulence intensities lower than $30 \%$. At the height of the boundary layer the Cobra are operating, $\sim 0.4 H_{r e f . F C 2}$, turbulence intensities are relatively high but lower than such limit. In addition, the proximity of the inner anemometers to the façades (the distance is approximately equal to $1 \mathrm{~cm}$ in model scale) might affect the accuracy of the measurements. However, such flow field study aims essentially to characterize qualitatively the flow within the gap.

Table 4 summarizes the most significant results, presented as the ratio between the mean of the velocity components and the mean reference velocity measured at $H_{r e f, F C 2}$; firstly, the percentage of good data acquired by the anemometer is shown to ensure that the anemometers are correctly oriented. Figure 17 shows the corresponding configurations. The velocity components $\mathrm{U}$ and $\mathrm{V}$ are defined on an ideal horizontal plane and the first one is associated with the direction along which the sensor is aligned to and $\mathrm{V}$ to the perpendicular one. Finally, the vertical velocity component $\mathrm{W}$ is also measured.

Let us first focus on the $-120^{\circ}$ configuration (Figure 17(a)): the $\mathrm{U}$ component is always higher than the others, since the sensors have been oriented according to the expected flow direction. By comparing the 


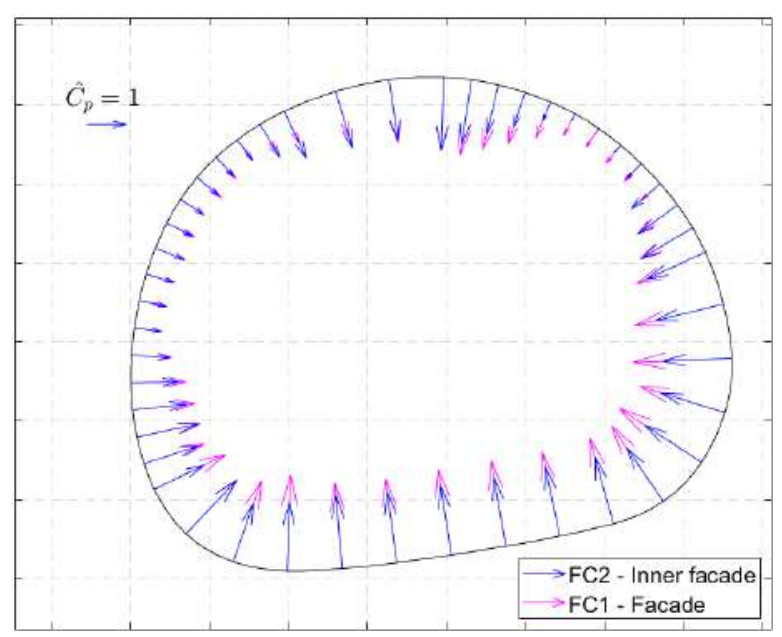

(a)

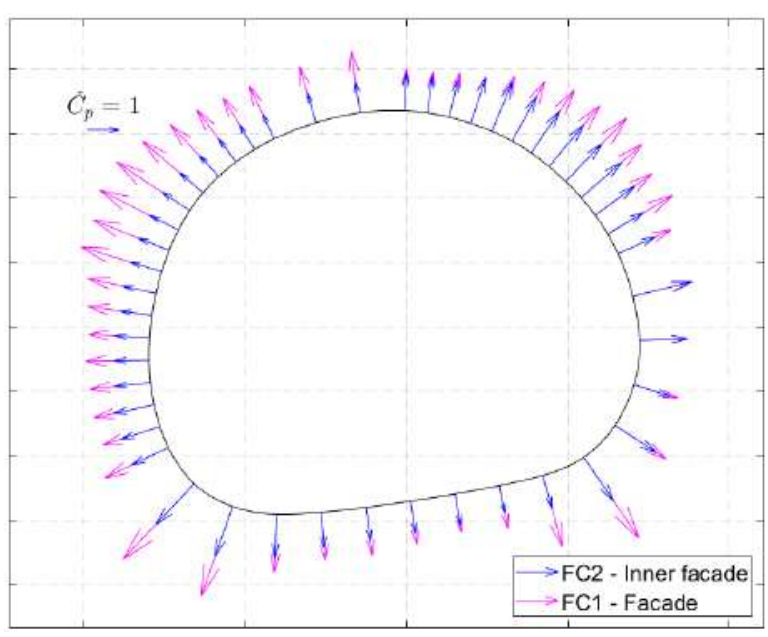

(b)

Figure 16: (a) $\hat{C}_{p}$ and (b) $\breve{C}_{p}$ envelope distributions for the glazed façade: $F C 1$ test and $F C 2$ test comparison.

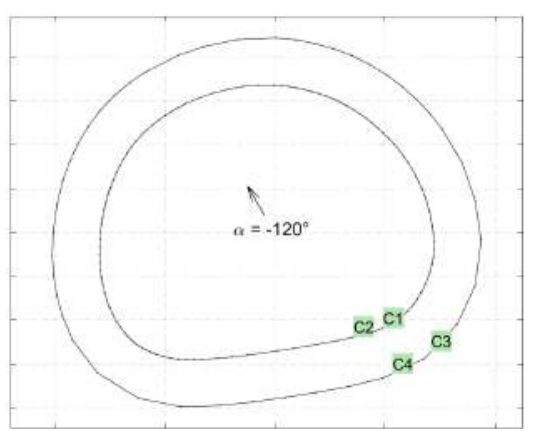

(a)

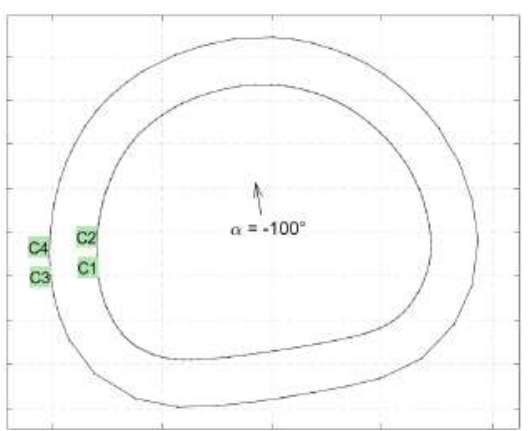

(b)

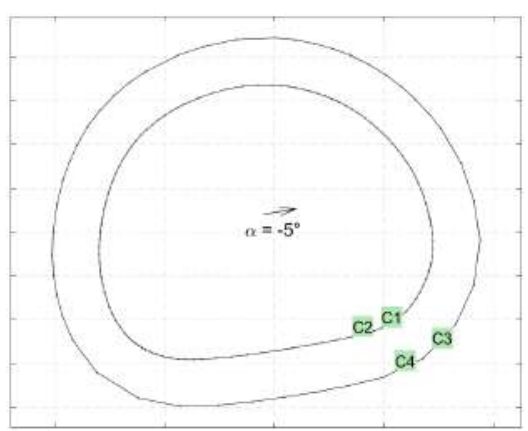

(c)

Figure 17: Cobra probes position for incoming wind directions (a) $-120^{\circ}$, (b) $-100^{\circ}$ and (c) $-5^{\circ}$.

outer anemometers to the ones placed in the gap between the façades, it can be noted that there is an intake with a drop in the $\mathrm{U}$ velocity component passing from outside to the inner gap.

Switching to the set up depicted in Figure 17(b), the anemometers have been placed such that they are parallel to the façade perimeter. We still have that the $\mathrm{U}$ component is dominant compared to $\mathrm{V}$ and $\mathrm{W}$ values, which are marginally contributing to the total flow velocity. Results confirm that we have flow running along an ideal line parallel to the inner façade while a negligible flow occurs along the outward normal with respect to the glazed skin.

Finally, referring to the $-5^{\circ}$ configuration (Figure $17(\mathrm{c})$ ), where the sensors have been oriented again according to the building perimeter, the $\mathrm{U}$ component acquired by the inner anemometers is halved with respect to the values outside the mesh.

\subsubsection{Time and frequency domain comparison}

The effect of the mesh on the acquired pressure signals is further addressed considering the wind direction $-140^{\circ}$, which is significant for both thrusts and suctions. To this purpose, pressure signals recorded on 


\begin{tabular}{cccccc}
\hline$\alpha$ & & $\mathrm{C} 1$ & $\mathrm{C} 2$ & $\mathrm{C} 3$ & $\mathrm{C} 4$ \\
\hline \multirow{4}{*}{$120^{\circ}$} & \% Good Data & 98 & 91 & 100 & 98 \\
& $\mathrm{U} / \mathrm{U}_{H_{\text {ref } F C 2}}$ & 0.66 & 0.49 & 1.18 & 0.97 \\
& $\mathrm{~V} / \mathrm{U}_{H_{\text {ref }, F 2}}$ & 0.04 & 0.02 & 0.37 & -0.04 \\
& $\mathrm{~W} / \mathrm{U}_{H_{\text {ref } F C 2}}$ & -0.10 & -0.08 & 0.04 & 0.01 \\
\hline \multirow{4}{*}{$100^{\circ}$} & $\%$ Good Data & 95 & 85 & 100 & 99 \\
& $\mathrm{U} / \mathrm{U}_{H_{\text {ref }, F C 2}}$ & 0.57 & 0.44 & 1.10 & 0.78 \\
& $\mathrm{~V} / \mathrm{U}_{H_{\text {ref }, F C 2}}$ & -0.03 & -0.04 & -0.01 & -0.01 \\
& $\mathrm{~W} / \mathrm{U}_{H_{\text {ref }, F C 2}}$ & 0.02 & 0.04 & 0.04 & 0.05 \\
\hline \multirow{4}{*}{$5^{\circ}$} & \% Good Data & 93 & 95 & 98 & 100 \\
& $\mathrm{U} / \mathrm{U}_{H_{\text {ref }, F C 2}}$ & 0.35 & 0.34 & 0.58 & 0.73 \\
& $\mathrm{~V} / \mathrm{U}_{H_{\text {ref }, F C 2}}$ & -0.08 & -0.06 & -0.03 & -0.10 \\
& $\mathrm{~W} / \mathrm{U}_{H_{\text {ref }, F C 2}}$ & -0.09 & -0.13 & -0.05 & -0.09 \\
\hline
\end{tabular}

Table 4: Cobra measurement results

the façade during the $F C 2$ stage are compared in both time and frequency domain to the $F C 1$ ones acquired in the corresponding points.

As an example in the thrust region, pressure tap in position T1, as depicted in Figure [18, is selected for $F C 1$ and $F C 2$. When considering the flow-detachment region, T2 sensor position for $F C 1$ and $F C 2$ is chosen. The time histories of the analyzed signals $C_{p}(t)$ are depicted in Figures 19(a) and 20(a) for positive and negative pressure cases, respectively. For the case at hands, the application of the mesh results in lower absolute mean value and standard deviation of the $F C 2$ pressure signals, e.g. the one acting on the inner façade. In fact, the sharp pressure spikes which are typically recorded on the standard façade, e.g. $F C 1$ test, are filtered by the metallic mesh and, thus, disappear on the inner façade. This is particularly relevant for the suction case, which is usually the most severe in terms of peak pressures. As reported in Table 5. the standard deviation of the $F C 2$ pressure signals halves. Being the probability density function (PDF) tails associated with the extreme value analysis (Lou and Peterka, 1981), the influence of the porous media is also studied in that regard. Figures 21(a) and 21(b) show the PDF of the same pressure signals for the thrust and suction cases, respectively. When comparing the $F C 1$ and $F C 2$, the difference in the tails of the distribution and thus in the degree of Gaussianity of the pressure signals is evident. As shown in Table 5, the skewness of $F C 2$ pressure signals is $\sim 40 \%$ lower than the ones estimated for the $F C 1$ case. This behaviour is due to the application of the porous skin, which effectively filters the oncoming turbulence and therefore determines the different dynamic content of pressure signals recorded on the inner façade during $F C 2$.

The power spectral density functions (PSD) of the pressure fluctuations analyzed above are depicted in Figures 19(b) and 20(b). As expected, the energy content of the $F C 1$ signal is higher than the corresponding one recorded on the inner façade within the entire frequency axes. In fact, spectra vary due to the nature of the physical processes that occur near the surfaces in the boundary layer flow and lead to different aerodynamic behavior in the two configurations due to the presence of the mesh.

\section{Reduction of the Peak Pressure Values}

\subsection{Reduction Factors}

As the peak pressure distributions are computed for both $F C 1$ and $F C 2$ systems, the reduction of the design wind loads for the inner façade of the DSF system can be assessed. Therefore, a reduction factor 


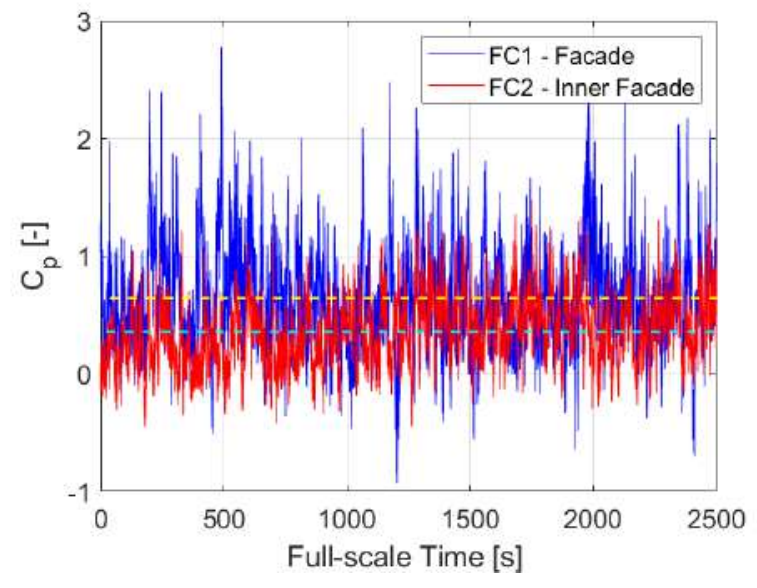

(a)

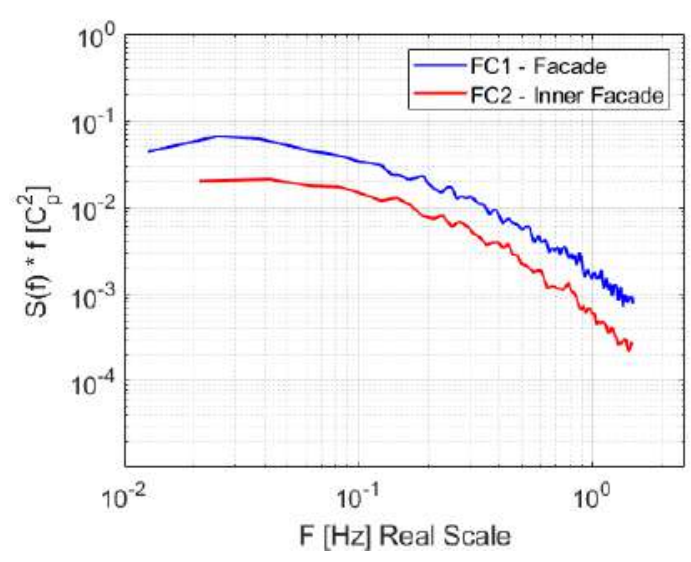

(b)

Figure 19: Example of $C_{p}$ (a) time history and (b) PSD for positive pressure signal, $-140^{\circ}$ wind direction.

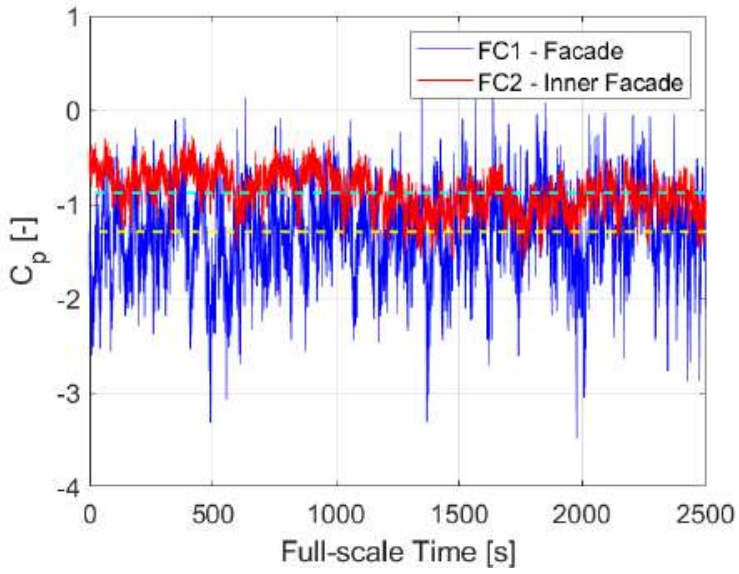

(a)

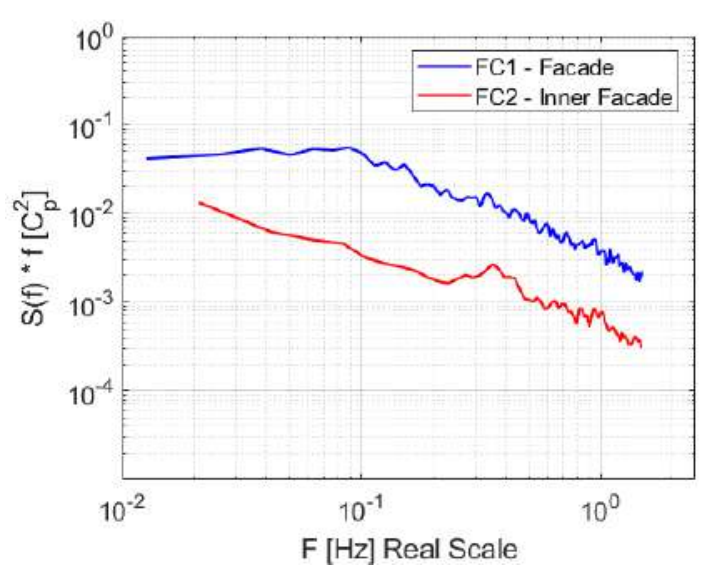

(b)

Figure 20: Example of $C_{p}$ (a) time history and (b) PSD for negative pressure signal, $-140^{\circ}$ wind direction. 
Table 5: Statistics of Pressure Time Series Data: FC1 and FC2 comparison.

\begin{tabular}{cccccc}
\hline Tap & Setup & Direction & Mean & Std & Skewness \\
\hline \multirow{2}{*}{ T1 } & $F C 1$ & $-140^{\circ}$ & 0.643 & 0.426 & 0.564 \\
& $F C 2$ & $-140^{\circ}$ & 0.360 & 0.277 & 0.298 \\
\hline \multirow{2}{*}{$\mathrm{T} 2$} & $F C 1$ & $-140^{\circ}$ & -1.293 & 0.443 & -0.346 \\
& $F C 2$ & $-140^{\circ}$ & -0.876 & 0.211 & -0.203 \\
\hline
\end{tabular}

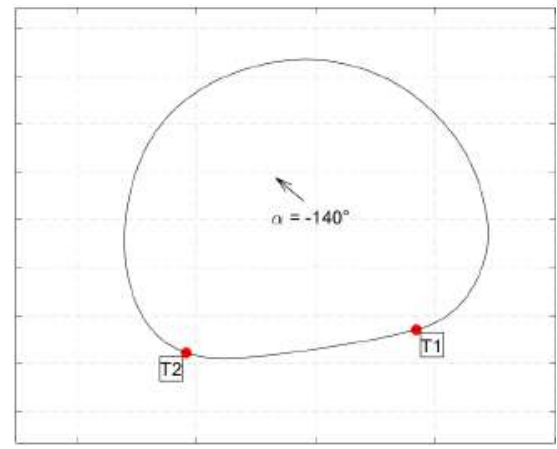

Figure 18: T1 and T2 pressure taps position.

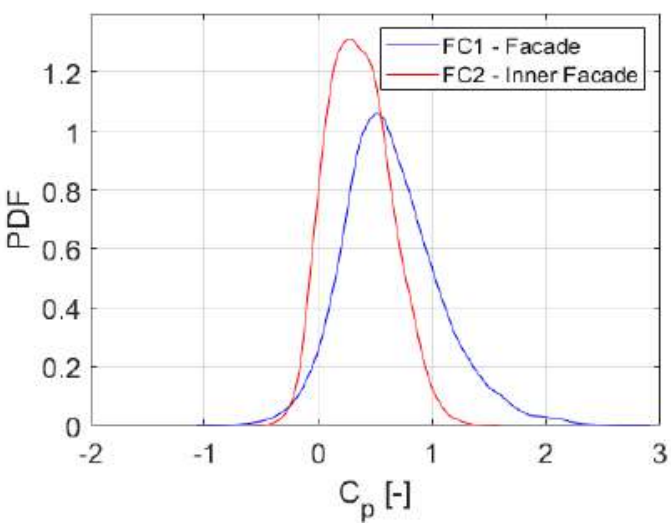

(a)

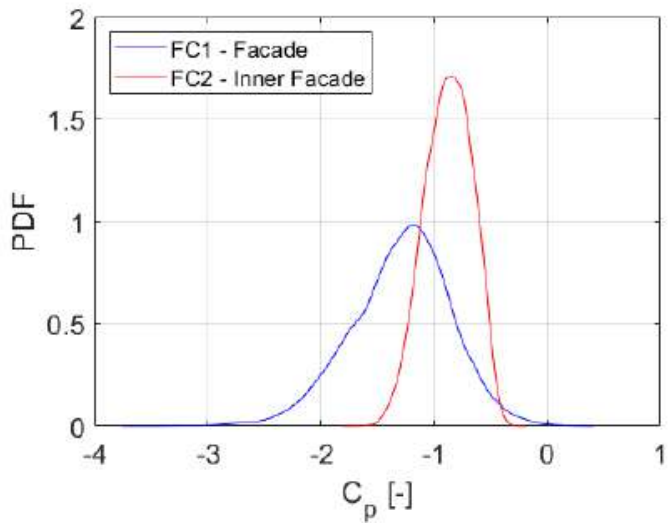

(b)

Figure 21: PDFs of signals which exhibit strong thrusts (a) and suctions (b): comparison between $F C 1$ test and $F C 2$ test.

is calculated based on the peak pressures obtained with the standard façade system, e.g. $F C 1$ stage, by considering the correspondence of the pressure taps locations during the two experimental tests. Making reference to both the positive $\left(\hat{C}_{p}\right)$ and negative $\left(\breve{C}_{p}\right)$ peak pressures, the reduction factors are defined, respectively, as:

$$
\hat{\eta}=\frac{\hat{C}_{p, F C 1}-\hat{C}_{p, F C 2}}{\hat{C}_{p, F C 1}}[\%] \quad \check{\eta}=\frac{\check{C}_{p, F C 1}-\check{C}_{p, F C 2}}{\check{C}_{p, F C 1}}[\%]
$$

Since the reduction factors are essential for the design peak pressure, we focus only on non-dimensional peak pressure coefficients $\hat{C}_{p, F C 1}>1.5$ and $\breve{C}_{p, F C 1}<-1.5$. The reduction factors corresponding to peak pressure coefficients outside such a range are set equal to zero. The $\hat{\eta}$ and $\check{\eta}$ factors are computed based on the positive and negative peak pressure envelopes (the most critical pressure value among all the incoming wind directions for each pressure tap) of the standard façade system. Figure 22 shows the calculated reduction factors. The decrease of peak pressures acting on the inner façade of the DSF system is remarkable for both positive and negative peak pressure envelopes. In addition, the highest values of the reduction factors are found in the regions which correspond to the most severe peak pressure estimated for the $F C 1$ case. In particular, the strongest suction of the $F C 1$ test is reduced by $63 \%$ if the DSF system is utilized: $\check{C}_{p}$ increases from -3.21 to -1.18 (Table 6). These findings, which further highlights the filtering features of the 
Table 6: Extreme values from envelopes of $F C 1$ test and corresponding reduction factors.

\begin{tabular}{lccc}
\hline & $F C 1$ & $F C 2$ & $\eta[\%]$ \\
\hline$\check{C}_{p}[-]$ & -3.214 & -1.189 & 63 \\
$\hat{C}_{p}[-]$ & 2.509 & 1.581 & 37 \\
\hline
\end{tabular}

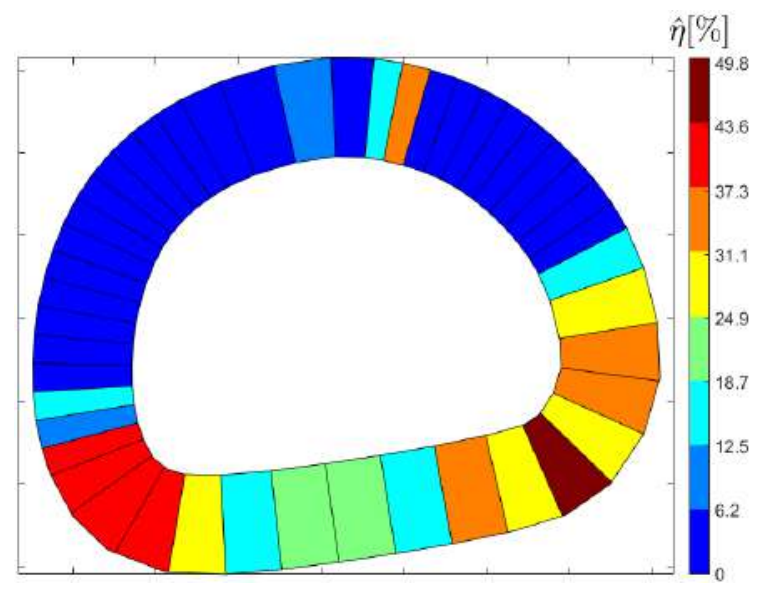

(a)

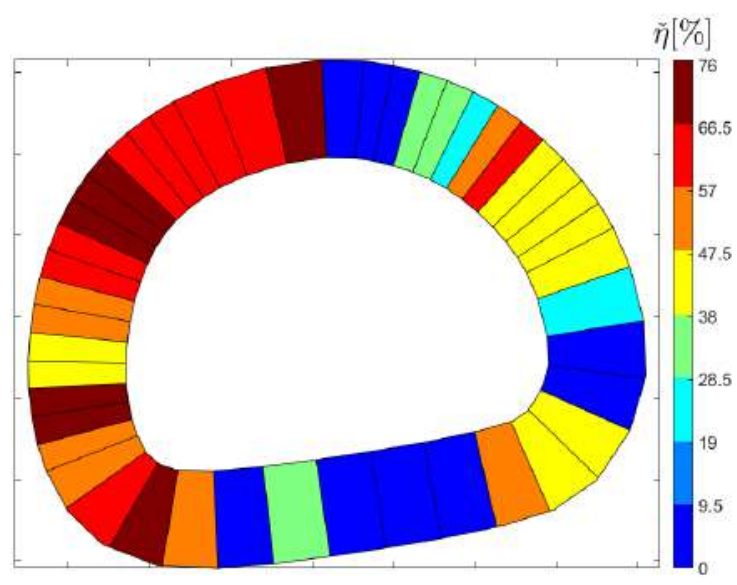

(b)

Figure 22: Reduction factors for the positive (a) and negative (b) pressure envelopes for the tested building.

mesh, are essential since the peak pressure envelopes are the design tool for the cladding wind loads.

\subsection{Generalization}

The computation of the reduction factors is extended to obtain a statistically significant population. To this purpose, for each pressure measurement point, the peak pressures computed for every wind direction are considered. The same lower bound of non-dimensional pressure coefficient mentioned above is utilized here. Figure 23 shows the computed reduction factor based on both positive and negative peak pressures estimated during the $F C 1$ test. The effect of the metallic mesh on the peak pressures computed for the inner façade is relevant. In particular, the higher the absolute value of the $F C 1$ peak pressure, the higher the reduction factors are. Hence, a lower bound can be arbitrarily set for the reduction factors of both positive and negative peak pressures, as depicted in Figure 23. The lower bound is chosen to be described by a straight line using the following expressions:

$$
\hat{\eta}=\left\{\begin{array}{ll}
0 & \text { if } \hat{C_{p}} \leq 1.90 \\
45\left(\hat{C}_{p}-1.90\right) & \text { if } \hat{C_{p}}>1.90
\end{array} \quad \check{\eta}= \begin{cases}0 & \text { if } \check{C_{p}} \geq-1.80 \\
-35\left(\check{C}_{p}+1.80\right) & \text { if } \check{C_{p}}<-1.80\end{cases}\right.
$$

The definition of a lower bound for the reduction factors aims to obtain a qualitative estimate on the safe side of the peak pressures expected on the inner façade of the DSF system. Figure 23 provides a relevant design tool for cladding loads, underpinning the prime importance of the wind tunnel tests for the DSF systems. 


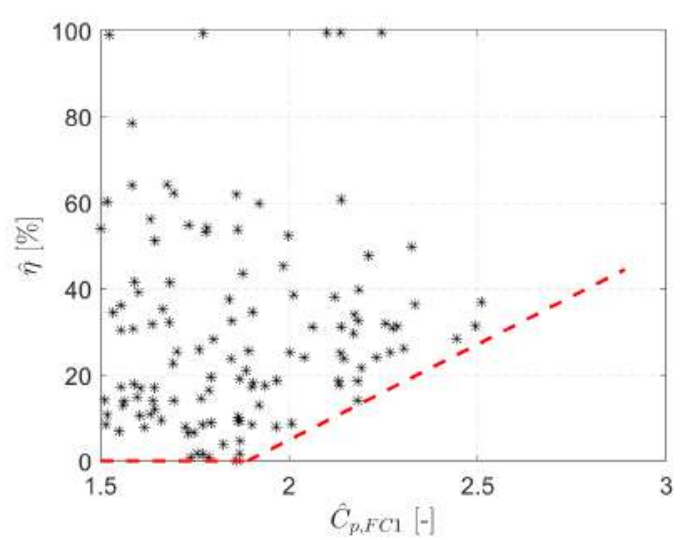

(a)

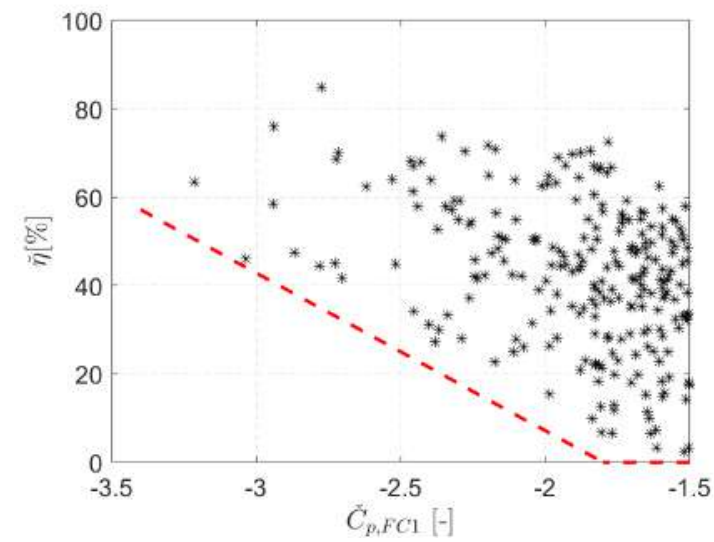

(b)

Figure 23: $F C 1$ test $\hat{C_{p}}$ (a) and $\breve{C_{p}}$ (b) and the corresponding reduction factors.

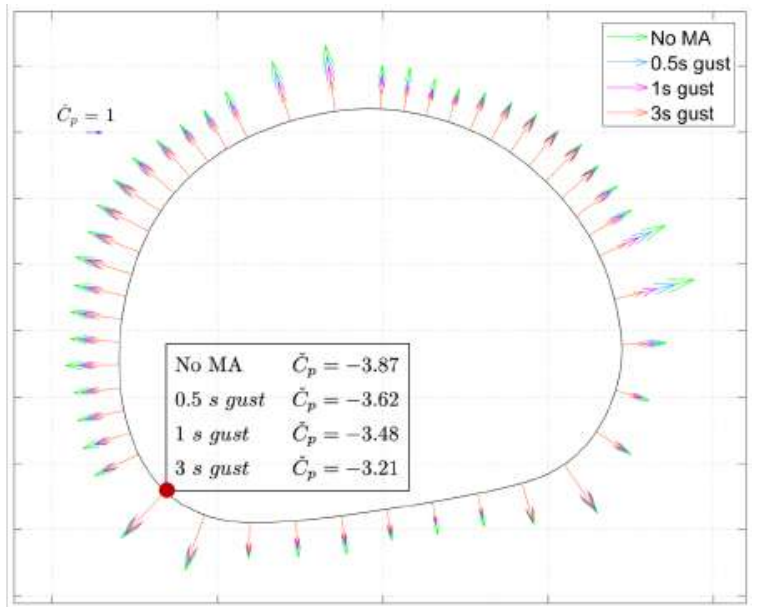

(a)

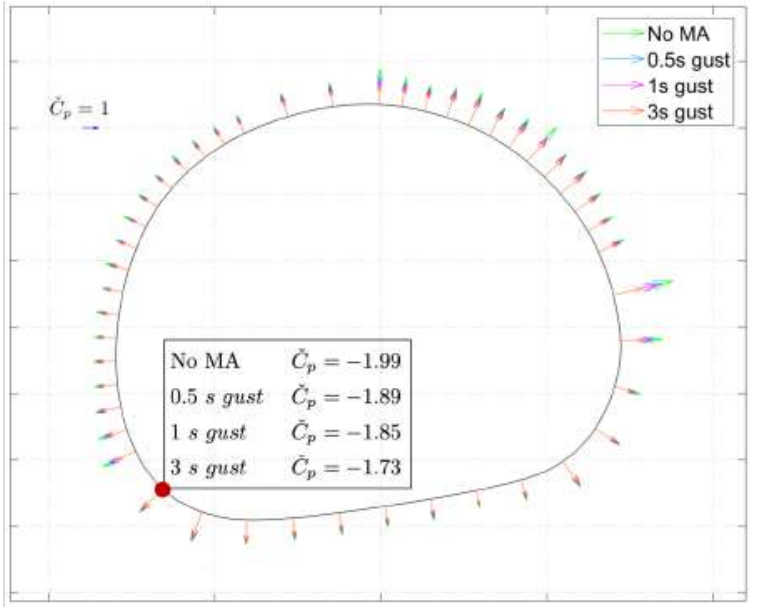

(b)

Figure 24: $\check{C_{p}}$ envelopes for the $F C 1$ (a) and the $F C 2$ (inner skin) (b) stages, for different values of the averaging time (no moving average filter, $0.5 \mathrm{~s}, 1 \mathrm{~s}$ and $3 \mathrm{~s}$ ). 


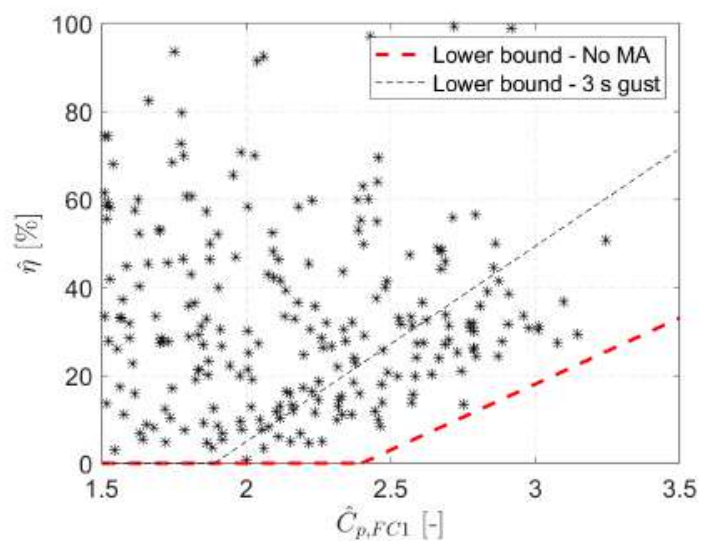

(a)

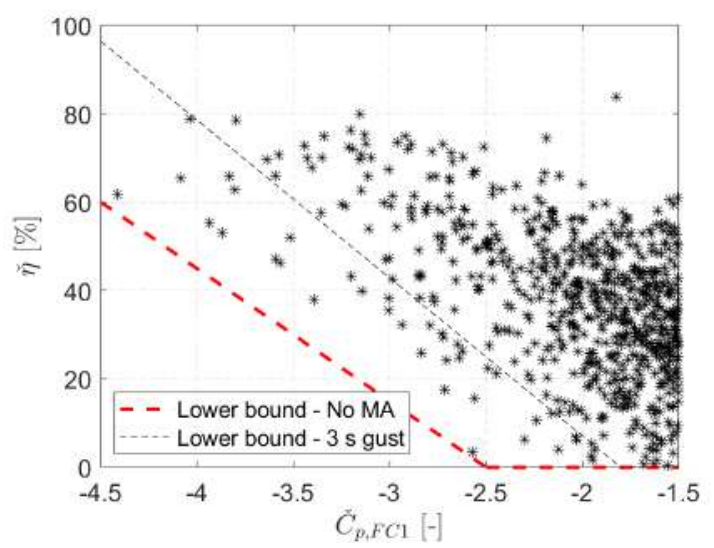

(b)

Figure 25: $F C 1$ test $\hat{C}_{p}$ (a) and $\check{C}_{p}$ (b) with no moving average filter and the corresponding reduction factors. The red dotted lines highlight the lower bound of the point cloud and the black dotted lines correspond to the same limit for the $3 \mathrm{~s}$ gust case.

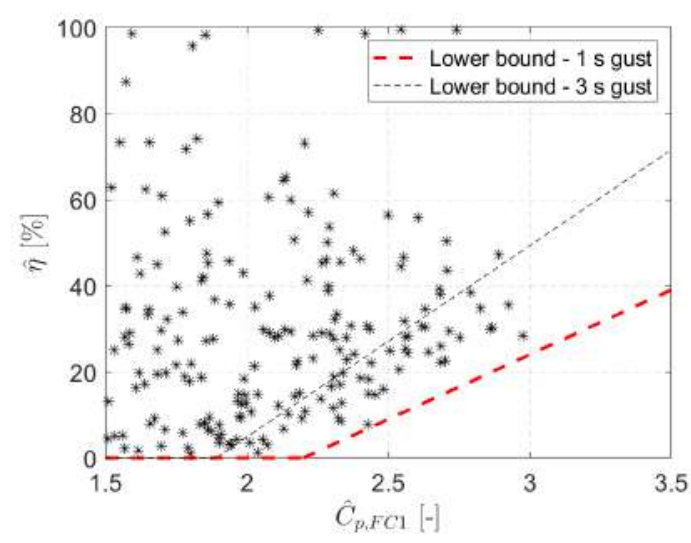

(a)

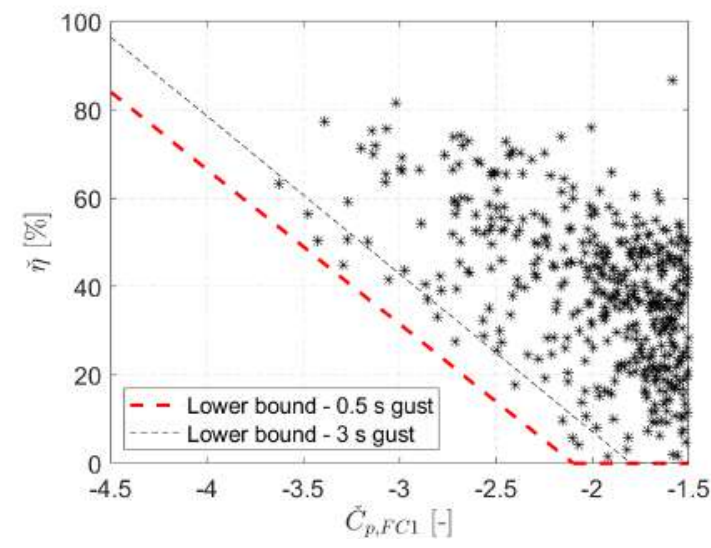

(b)

Figure 26: $F C 1$ test $\hat{C_{p}}$ (a) and $\breve{C_{p}}$ (b) and the corresponding reduction factors for the $0.5 s$ gust case. The red dotted lines highlight the lower bound of the point cloud and the black dotted lines correspond to the same limit for the $3 \mathrm{~s}$ gust case. 


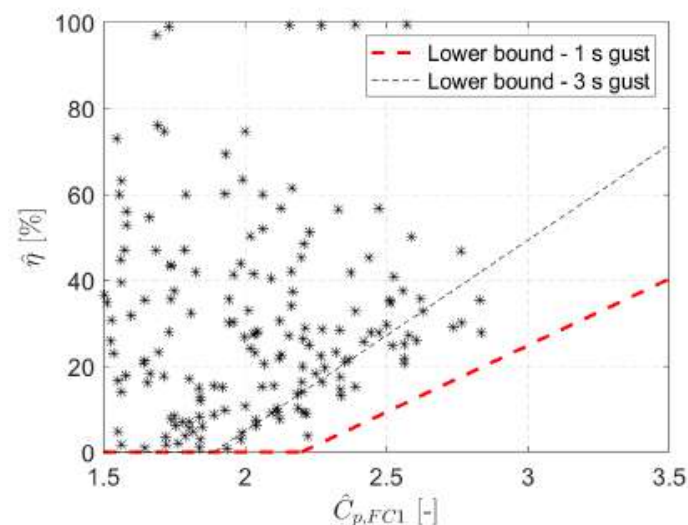

(a)

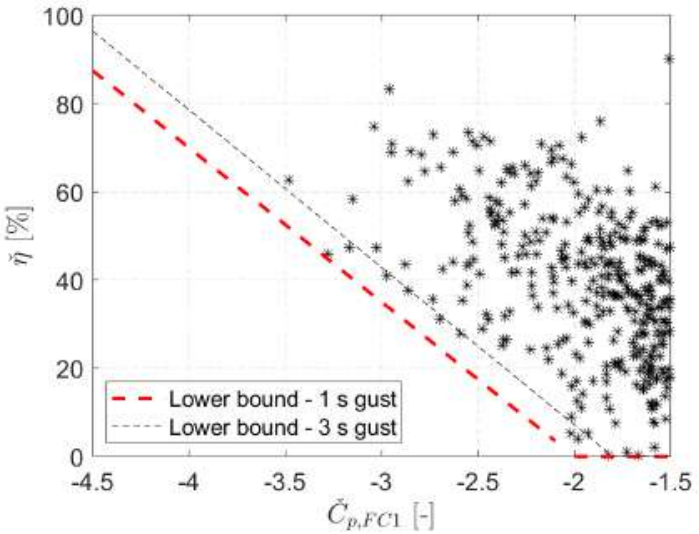

(b)

Figure 27: $F C 1$ test $\hat{C}_{p}$ (a) and $\breve{C}_{p}$ (b) and the corresponding reduction factors for the $1 s$ gust case. The red dotted lines highlight the lower bound of the point cloud and the black dotted lines correspond to the same limit for the $3 s$ gust case.

\subsection{Remarks on the 3 s gust}

All the extreme values analysis results presented up to this point come from the post-processing of the acquired pressure time series filtered by a moving average operator with an averaging time of $3 \mathrm{~s}$ real scale. This filter is commonly used in the wind tunnel current practice (Amerio, 2018) to account for the lack of simultaneous correlation in space of pressure signals.

Further investigations about the effects of the choice of the averaging time on the peak pressure distributions and the envelope diagrams have been performed, since the existing literature declares a lack of a fully justified procedure in the determination of the most suitable averaging time (Holmes, 1997) (Amerio, 2018). At this aim, in addition to the $3 \mathrm{~s}$ gust, different averaging times have been selected, i. e. $1 \mathrm{~s}, 0.5 \mathrm{~s}$ and no averaging at all.

The analyses previously shown have highlighted how the presence of the outer skin modifies the peak pressure distributions and the flow conditions on the inner glazed skin with respect to the standard façade case. It is shown that signals from the $F C 1$ have a higher dynamic content, with spikes greater in amplitude than the ones from the $F C 2$ signals. This different "nature" reflects on a different effect of the moving mean operator: pressure signals from the $F C 1$ test are much more affected by the operator than the ones from the inner façade of the $F C 2$ stage. This impacts on the estimation of the extreme values: by applying Cook and Mayne method to the same signal, previously filtered by the moving mean operator with different averaging times, the resulting $C_{p}$ peaks are quite different. As shown by the envelope diagrams depicted in figure 24(a) changing the averaging time (no filter, $0.5 \mathrm{~s}, 1 \mathrm{~s}, 3 \mathrm{~s}$ ), the difference in length is appreciable and the longest arrows are more than halved if the $3 \mathrm{sec}$ gust is applied with respect to the extreme values corresponding to the no averaged signals. The text in Figure 24(a) reports the $\check{C}_{p}$ values for the tap that experiences one of the greatest suctions: the difference switching from the first and the last filter (i.e. No MA and $3 \mathrm{~s}$ gust) is about $0.6 C_{p}$. Figure 24(b) reports the same plot for the inner facade $C_{p}$ negative peak values: here, switching from the green to the red arrows, the length reduction is less evident. Focusing on the tap marked in red, the negative peak pressure coefficient is increased by $0.26 C_{p}$ applying the $3 \mathrm{~s}$ averaging time with respect to the raw acquired signal. The smaller effect of the averaging filter on the FC2 extreme values is mainly due to the fact that those signals have been affected by the mesh effect and already "smoothed" by it.

The different effect of the choice of the averaging time on the peak values has a direct impact also 
on the reduction factors, that have been re-calculated for all the signals treated with the different filters. Figures from 25 to 27 show the points clouds for positive and negative peak pressures, computed varying the averaging time. The dashed red line highlights the lower bound of the cloud and the black dashed line the limit associated with the $3 \mathrm{~s}$ gust filter. For sake of completeness, we list the expressions of the lower bounds, varying the averaging time:

- if no moving average filter is applied:

$$
\hat{\eta}=\left\{\begin{array}{ll}
0 & \text { if } \hat{C}_{p} \leq 2.40 \\
30\left(\hat{C}_{p}-2.40\right) & \text { if } \hat{C}_{p}>2.40
\end{array} \quad \check{\eta}= \begin{cases}0 & \text { if } \check{C}_{p} \geq-2.50 \\
-30\left(\check{C}_{p}+2.50\right) & \text { if } \check{C}_{p}<-2.50\end{cases}\right.
$$

- for the $0.5 s$ gust case:

$$
\hat{\eta}=\left\{\begin{array}{ll}
0 & \text { if } \hat{C_{p}} \leq 2.20 \\
30\left(\hat{C}_{p}-2.20\right) & \text { if } \hat{C}_{p}>2.20
\end{array} \quad \check{\eta}= \begin{cases}0 & \text { if } \check{C}_{p} \geq-2.10 \\
-35\left(\check{C}_{p}+2.10\right) & \text { if } \check{C}_{p}<-2.10\end{cases}\right.
$$

- for the 1 s gust case:

$$
\hat{\eta}=\left\{\begin{array}{ll}
0 & \text { if } \hat{C_{p}} \leq 2.20 \\
31\left(\hat{C_{p}}-2.20\right) & \text { if } \hat{C_{p}}>2.20
\end{array} \quad \check{\eta}= \begin{cases}0 & \text { if } \check{C_{p}} \geq-2.00 \\
-35\left(\check{C}_{p}+2.00\right) & \text { if } \check{C}_{p}<-2.00\end{cases}\right.
$$

Decreasing the averaging time, it appears that the lower bound is horizontally shifted toward higher (in module) peak pressures, which means that for the same $\check{C}_{p, F C 1}$ or $\hat{C}_{p, F C 1}$, a lower value of $\eta$ than the one corresponding to the $3 \mathrm{~s}$ gust filter should be considered for the design of the inner façade of a DSF system.

\section{Conclusions}

The paper presents the experimental methodologies utilized during the wind tunnel tests to assess the cladding loads for a porous double skin façade (DSF). Using the low-rise buildings of New Bocconi Campus as a case study, a multi-scale approach is adopted to investigate the aerodynamic effects of the porosity on the pressure distribution on the inner façade of the DSF system. In particular, the reduction of the peak pressures, which are relevant for the cladding design, is addressed, leading to the following findings for the case at hands:

- Both the positive and negative peak pressures estimated on the inner façade are found to be up to $40 \%$ lower than the corresponding one for standard façade system. In addition, the largest decreases of the peak pressures acting on the inner façade of the DSF system studied correspond to the most severe peak pressures estimated when testing the standard façade system. For example, the strongest suction which dictates the cladding design for the latter case is reduced by $63 \%$ ( $\check{C}_{p}$ increases from -3.21 to -1.18) with the adoption of the porous DSF system designed for the low-rise buildings analyzed. This finding is essential since the cladding design is generally dictated by a unique value.

- For the case at hands, additional investigations have been addressed, i.e. velocity measurements and pressure signals comparison. The former have highlighted the presence of a flow within the gap between the two façades. The latter has allowed to remark the aerodynamic effects of the porous skin on the pressure signals acquired on the inner façade are remarkable. In fact, both the absolute mean 
values and standard deviations of the pressure signals are found to be generally lower when testing the porous DSF system. Such a filtering effect of the mesh is particularly evident for the suctions considering the most severe wind direction, where the standard deviation of the recorded pressures is halved. The lower skewness estimated for the inner façade pressures, which is relevant for the extreme value distribution, is also highlighted. Spectra comparison shows a lower energy content for the inner skin pressure signals with respect to the one acquired in the standard façade case.

- The higher the peak pressures estimated in each pressure measurement point for the standard façade case considering every wind direction, the higher the corresponding reductions are.

- The dependency of the extreme values of pressures on the averaging time is more evident for the standard façade case for the case at hands. This reflects the filtering effect of the porous skin on inner façade pressure signals, which, thus, exhibit a higher correlation in space.

\section{References}

AFNOR, 2008. Eurocode 1: actions on structures - part 1:4: General actions - wind actions.

Aly, A. M., Bitsuamlak, G., 2013. Aerodynamics of ground-mounted solar panels: test model scale effects. Journal of Wind Engineering and Industrial Aerodynamics 123, 250-260.

Amerio, L., 2018. Experimental high resolution analysis of the pressure peaks on a building scale model facades. Ph.D. thesis, Italy.

Belloli, M., Muggiasca, S., Rosa, L., Zasso, A., 2016. Experimental study on the aerodynamic behavior of a circular cylinder with perforated shrouding. In: 8th International Symposium on Bluff Body Wakes and Vortex-Induced Vibrations. pp. 1-9.

Belloli, M., Rocchi, D., Rosa, L., Zasso, A., 2012. Wind tunnel studies on the effects of porous elements on the aerodynamic behavior of civil structures. In: The Seventh International Colloquium on Bluff Body Aerodynamics and Applications (BBAA7). pp. 1132-1141.

Belloli, M., Rosa, L., Zasso, A., 2014. Wind loads and vortex shedding analysis on the effects of the porosity on a high slender tower. Journal of Wind Engineering and Industrial Aerodynamics 126, 75-86.

Bergh, H., Tijdeman, H., 1965. Theoretical and experimental results for the dynamic response of pressure measuring systems.

Cook, N., Mayne, J., 1979. A novel working approach to the assessment of wind loads for equivalent static design. Journal of Wind Engineering and Industrial Aerodynamics 4 (2), 149-164.

Cook, N. J., 1986. Designers guide to wind loading of building structures. part 1.

da Silva, F. M., Gomes, M. G., 2008. Gap inner pressures in multi-storey double skin facades. Energy and Buildings 40 (8), 1553-1559.

Diana, G., Fiammenghi, G., Belloli, M., Rocchi, D., 2013. Wind tunnel tests and numerical approach for long span bridges: the messina bridge. Journal of Wind Engineering and Industrial Aerodynamics 122, 38-49.

Gerhardt, H., Janser, F., 1994. Wind loads on wind permeable facades. Journal of Wind Engineering and Industrial Aerodynamics 53 (1-2), 37-48.

Gerhardt, H., Kruger, O., 1997. Double skin glass facades-investigations into the load sharing possibilities. Proc. of ICBEST 97, $335-339$.

Holmes, J. D., 1997. Equivalent time averaging in wind engineering. Journal of Wind Engineering and Industrial Aerodynamics 72, 411-419.

Hu, G., Hassanli, S., Kwok, K. C., Tse, K.-T., 2017. Wind-induced responses of a tall building with a double-skin façade system. Journal of Wind Engineering and Industrial Aerodynamics 168, 91-100.

Kemper, F., Feldmann, M., 2019. Wind load assumptions for permeable cladding elements considering the installation context. Journal of Wind Engineering and Industrial Aerodynamics 184, 277-288.

Kilpatrick, J., Crosbie, E., Jain, A., Kumar, S., 2009. Load sharing on double skin façades.

Letchford, C., Row, A., Vitale, A., Wolbers, J., 2000. Mean wind loads on porous canopy roofs. Journal of Wind Engineering and Industrial Aerodynamics 84 (2), 197-213.

Lou, J.-J., Peterka, J. A., 1981. Extreme value analysis of peak wind pressures on buildings. CER; 81/82-32.

Mayne, J., Cook, N., 1980. Acquisition, analysis and application of wind loading data. In: Wind Engineering. Elsevier, pp. 13391355.

Moon, K. S., 2011. Structural design of double skin facades as damping devices for tall buildings. Procedia Engineering 14, 1351-1358. 
Overend, M., Zammit, K., 2006. Wind loading on cladding and glazed façades. In: Proceedings.

Poizaris, H., 2004. Double skin facades for office buildings: literature review. Tech. rep., Report EBD.

Xiaotong, P., Chen, L., 2010. Cfd modeling for thermal behavior of open-type double-skin facades. In: Intelligent Computation Technology and Automation (ICICTA), 2010 International Conference on. Vol. 1. IEEE, pp. 918-921. 